\title{
Faceting and coarsening dynamics in the complex Swift-Hohenberg equation
}

\author{
Lendert Gelens, ${ }^{1, *}$ and Edgar Knobloch ${ }^{2}$ \\ ${ }^{1}$ Department of Applied Physics and Photonics, Vrije Universiteit Brussel, Pleinlaan 2, B-1050 Brussel, Belgium \\ ${ }^{2}$ Department of Physics, University of California, Berkeley, California 94720, USA
}

(Received 20 July 2009; published 30 October 2009)

\begin{abstract}
The complex Swift-Hohenberg equation models pattern formation arising from an oscillatory instability with a finite wave number at onset and finds applications in lasers, optical parametric oscillators, and photorefractive oscillators. We show that with real coefficients this equation exhibits two classes of localized states: localized in amplitude only or localized in both amplitude and phase. The latter are associated with phase-winding states in which the real and imaginary parts of the order parameter oscillate periodically but with a constant phase difference between them. The localized states take the form of defects connecting phase-winding states with equal and opposite phase lag, and can be stable over a wide range of parameters. The formation of these defects leads to faceting of states with initially spatially uniform phase. Depending on parameters these facets may either coarsen indefinitely, as described by a Cahn-Hilliard equation, or the coarsening ceases leading to a frozen faceted structure.
\end{abstract}

DOI: 10.1103/PhysRevE.80.046221

PACS number(s): 05.45.-a, 47.54.-r, 47.55.D-

\section{INTRODUCTION}

The real Swift-Hohenberg equation (RSHE) has proved to be an invaluable model equation for systems undergoing a bifurcation to time-independent structured states with a finite wave number at onset [1]. The equation was originally suggested as a model of infinite Prandtl number convection [2] but finds application in its simplest form in the theory of buckling [3], phase transitions [4], and nonlinear optics [5,6]. The equation is particularly useful in understanding localized structures that are commonly found in systems exhibiting bistability between two states, one of which is homogeneous in space (the trivial state) while the other is heterogeneous or structured [1]. In this case the equation takes the form, in one spatial dimension,

$$
u_{t}=r u-\left(\partial_{x}^{2}+k_{0}^{2}\right)^{2} u+f(u)
$$

where $u(x, t)$ is a real order parameter and $f(u)$ denotes nonlinear terms. Most useful are the two cases $f=f_{23} \equiv b_{2} u^{2}$ $-u^{3}$ [7] and $f=f_{35} \equiv b_{3} u^{3}-u^{5}$ [8]. When $b_{2}>\sqrt{27 / 38} k_{0}^{2}$ (respectively, $\left.b_{3}>0\right)$ the primary bifurcation from the trivial state $u=0$ takes place at $r=0$ and yields a subcritical branch of spatially periodic states with wave number $k_{0}$. This branch is unstable but acquires stability at finite amplitude at a saddle-node ( $\mathrm{SN}$ ) bifurcation. In addition, it is known that on the real line there are two (respectively, four) branches of spatially localized states (LSs) that bifurcate from $u=0$ simultaneously with the periodic states, and do so likewise subcritically. These states are therefore also initially unstable. When followed numerically these states become better and better localized and once their amplitude and width become comparable to the amplitude and wavelength of the competing periodic state these states begin to grow in spatial extent by adding rolls symmetrically at either side. In a bifurcation diagram this growth is associated with back and forth oscillations across a "pinning" interval. This behavior

\footnotetext{
*lendert.gelens@vub.ac.be
}

is known as homoclinic snaking [1,7,9-11] and is associated with repeated gain and loss of stability of the associated LSs. On a qualitative level this behavior can be understood to be the result of pinning of the fronts at either side of the localized structure to the heterogeneous structure between them [12]. Mathematically, the snaking behavior is a consequence of transverse intersection of the two-dimensional unstable manifold of the homogeneous state and the threedimensional center-stable manifold of the spatially periodic state $[1,9,13,14]$, together with reversibility under $x \rightarrow-x$. These results are reviewed in [11].

There are many systems, however, that are described by the Swift-Hohenberg equation for a complex order parameter. This equation models pattern formation arising from an oscillatory instability with a finite wave number at onset $[15,16]$. As a result the complex Swift-Hohenberg equation (CSHE) frequently arises in nonlinear optics. For example, Lega et al. $[17,18]$ showed that the general set of MaxwellBloch equations for class $\mathrm{A}$ and $\mathrm{C}$ lasers can be adequately described by the CSHE; see also [19]. The CSHE also describes nondegenerate optical parametric oscillators (OPOs) [20-22], photorefractive oscillators [23], semiconductor lasers [24], and passively mode-locked lasers [25]. In general, the resulting Swift-Hohenberg equation in these systems has complex coefficients and hence time-dependent solutions. In the present paper we restrict attention to a special case of the CSHE, namely, the case of real coefficients, that continues to admit stationary solutions. Although this case admits all the time-independent solutions familiar from RSHE, it is a much richer system: since the real and imaginary parts of the order parameter are coupled by the nonlinear terms, the RSHE stationary states have different stability properties, and indeed the stationary CSHE solutions have nontrivial spatial structure in both amplitude and phase. Understanding of this special case is thus a prerequisite for gaining a deeper insight into the behavior of the CSHE with complex coefficients.

In the following section we describe the basic properties of the resulting equation, focusing on the bistable regime. In Sec. III we discuss the presence and stability of the so-called phase-winding states in which the real and imaginary parts 
of the order parameter oscillate with a constant but nonzero phase difference and the phase of each jumps by the same integer multiple of $2 \pi$ across the domain. In Sec. IV, we demonstrate numerically that localized states can take the form of defects connecting phase-winding states with equal and opposite phase lag. We study the bifurcation behavior of these LSs and show that they can be stable over a wide range of parameter values. In Sec. V we show that states with initially uniform phase are unstable to a faceting instability generating these defects and show that under appropriate conditions the growth of this instability is described by the Cahn-Hilliard equation for the spatial phase gradient. The predicted coarsening compares well with direct numerical simulations of the original complex Swift-Hohenberg equation. In other regimes we find that coarsening ceases when the LSs lock to their oscillatory tails; the computed transition between the coarsening and frozen regimes agrees well with numerical simulations. The paper concludes with a brief discussion.

\section{CUBIC-QUINTIC COMPLEX SWIFT-HOHENBERG EQUATION WITH REAL COEFFICIENTS}

We study the bistable CSHE with $f_{35}$ nonlinearity,

$$
u_{t}=r u-\left(\partial_{x}^{2}+k_{0}^{2}\right)^{2} u+b_{3}|u|^{2} u-|u|^{4} u,
$$

where $u$ is a complex field. The equation is fully parametrized by the real parameters $r$ and $b_{3}$, as well as the wave number $k_{0}$ or equivalently the domain length $L$; in the following we focus on a typical value of $b_{3}\left(b_{3}=1.5\right)$ and study the behavior of this equation as a function of the parameter $r$ for different choices of $k_{0}$ and $L$, as convenient.

We rewrite Eq. (2) in terms of either the real and imaginary parts of the order parameter $u(x, t)$, or in terms of amplitude and phase variables, defined by

$$
u(x, t)=u_{R}(x, t)+i u_{I}(x, t) \equiv R(x, t) e^{i \phi(x, t)} .
$$

Thus,

$$
\begin{gathered}
R_{t}=r R+b_{3} R^{3}-R^{5}-2 k_{0}^{2} R_{x x}+6 R_{x x} \phi_{x}^{2}+12 R_{x} \phi_{x} \phi_{x x}-k_{0}^{4} R \\
-R \phi_{x}^{4}+2 k_{0}^{2} R \phi_{x}^{2}+3 R \phi_{x x}^{2}+4 R \phi_{x} \phi_{x x x}-R_{x x x x} \\
R \phi_{t}=4 R_{x} \phi_{x}^{3}-6 R_{x x} \phi_{x x}-4 R_{x} \phi_{x x x}-4 k_{0}^{2} R_{x} \phi_{x}-4 R_{x x x} \phi_{x} \\
\quad+6 R \phi_{x}^{2} \phi_{x x}-2 k_{0}^{2} R \phi_{x x}-R \phi_{x x x x} .
\end{gathered}
$$

Spatially homogeneous solutions, hereafter referred to as "flat," take the form $u=R_{0}(t) e^{i \phi_{0}(t)}$. Thus,

$$
\begin{gathered}
R_{0 t}=\left(r-k_{0}^{4}\right) R_{0}+b_{3} R_{0}^{3}-R_{0}^{5}, \\
\phi_{0 t}=0,
\end{gathered}
$$

with stationary solutions given by

$$
\begin{gathered}
R_{0}^{2}=R_{s}^{2} \equiv\left\{0,\left[\frac{b_{3} \pm \sqrt{b_{3}^{2}+4\left(r-k_{0}^{4}\right)}}{2}\right]\right\}, \\
\phi_{0}=\phi_{s},
\end{gathered}
$$

where $\phi_{s}$ is any constant $\left(R_{s} \neq 0\right)$. The amplitude $R_{s}$ of these homogeneous states is shown in Fig. 1.

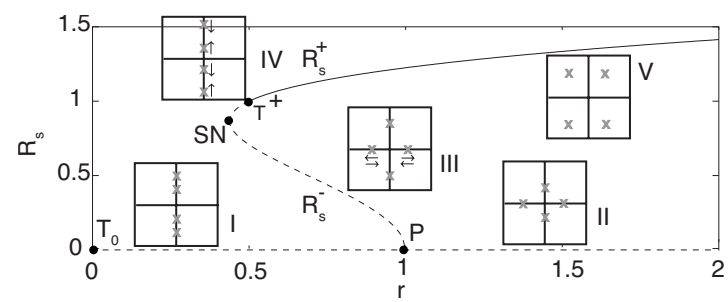

FIG. 1. Stationary homogeneous solutions $R_{s}$ for $b_{3}=1.5$ and $k_{0}=1$. These states are always unstable to phase modes, although the amplitude mode becomes stable beyond the Turing instability $T^{+}$of the nonzero state (solid line). Insets show the structure of the spatial eigenvalues of the amplitude mode along $R_{s}=0$ and $R_{s}^{ \pm} \neq 0$, as described by Eq. (15).

\section{A. Temporal stability}

To determine the stability of these states we let $u(x, t)$ $=\left[R_{s}+\delta(x, t)\right] \exp i \phi_{s}$, where $\delta$ is a complex infinitesimal perturbation satisfying

$$
\delta_{t}=r \delta-\left(\partial_{x}^{2}+k_{0}^{2}\right)^{2} \delta+b_{3} R_{s}^{2}(2 \delta+\bar{\delta})-R_{s}^{4}(3 \delta+2 \bar{\delta}) .
$$

Writing $\delta \equiv \delta_{R}+i \delta_{I}$ we obtain

$$
\begin{gathered}
\delta_{R t}=r \delta_{R}-\left(\partial_{x}^{2}+k_{0}^{2}\right)^{2} \delta_{R}+3 b_{3} R_{s}^{2} \delta_{R}-5 R_{s}^{4} \delta_{R}, \\
\delta_{I t}=r \delta_{I}-\left(\partial_{x}^{2}+k_{0}^{2}\right)^{2} \delta_{I}+b_{3} R_{s}^{2} \delta_{I}-R_{s}^{4} \delta_{I} .
\end{gathered}
$$

Thus, with $\left(\delta_{R}, \delta_{I}\right) \propto \exp (i k x+\sigma t)$ we find the pair of growth rates

$$
\begin{gathered}
\sigma_{R}=2 b_{3} R_{s}^{2}-4 R_{s}^{4}+\left(2 k_{0}^{2}-k^{2}\right) k^{2}, \\
\sigma_{I}=\left(2 k_{0}^{2}-k^{2}\right) k^{2},
\end{gathered}
$$

describing the stability of the flat states $R_{s}^{ \pm} \neq 0$ with respect to amplitude and phase perturbations, respectively.

Temporal stability of the trivial state. The state $u=0$ is destabilized by periodic modulations with wave number $k$ $=k_{0}$ at $r=0$ (point $T_{0}$ ) in a Turing bifurcation (also called modulational instability in the optics literature). A band of unstable wave numbers develops around $k=k_{0}$ for $r>0$ and spreads to $k=0$ when $r$ reaches $r=k_{0}^{4}$ (point $P$ ) at which point a Hamiltonian pitchfork bifurcation to nonzero flat states occurs (Fig. 1).

Temporal stability of the nonzero flat states. The state $R_{s}^{-}$ is always unstable with respect to amplitude perturbations. At the saddle-node bifurcation, $\left(r, u_{s}\right)=\left(-b_{3}^{2} / 4\right.$ $+k_{0}^{4}, \pm \sqrt{b_{3} / 2}$ ) (point SN), the $k=0$ amplitude mode stabilizes but a band of unstable wave numbers around $k=k_{0}$ remains. The last of these, $k=k_{0}$, stabilizes at $r=(1 / 8)\left(-b_{3}^{2}\right.$ $+10 k_{0}^{4}-b_{3} \sqrt{b_{3}^{2}+4 k_{0}^{4}}$ ) (point $T^{+}$) and for larger $r$ the upper branch $R_{s}^{+}$is stable with respect to amplitude modes (Figs. 1 and 2). In addition, the nonzero flat states are always unstable to phase perturbations with wave number $k$ in the range $0<k^{2}<2 k_{0}^{2}$; translation invariance implies that the phase growth rate of these perturbations vanishes when $k$ $=0$. Thus, the states beyond point $T^{+}$are amplitude stable but phase unstable, and for small wave numbers $\left(k \ll k_{0}\right)$ the growth rate of the instability is positive but small. This observation will be important in what follows. 


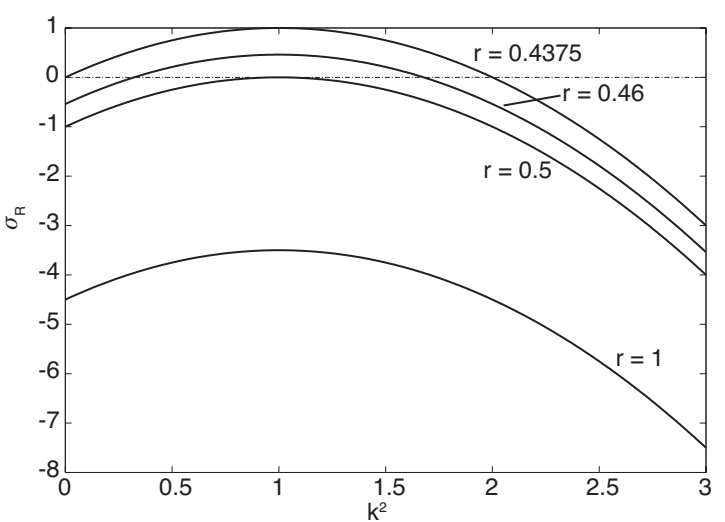

FIG. 2. Temporal growth rate $\sigma_{R}$ of the amplitude mode of the upper flat states $\left(R_{0}=R_{s}^{+}\right)$when $b_{3}=1.5$ and $k_{0}=1$ as given by Eq. (13).

Figure 1 summarizes these results. Solid (dashed) lines denote solutions that are stable (unstable) with respect to the amplitude mode. The figure also shows the spatial eigenvalues of the amplitude mode along the different branches.

\section{B. Spatial stability}

In order to understand the presence of LS homoclinic or heteroclinic to the flat states we also need to know their stability properties in space. For this purpose we write $R(x)=\left[R_{s}+\delta(x)\right] \exp i \phi_{s}$, where $\delta \equiv \delta_{R}+i \delta_{I} \propto e^{\lambda x}$. The spatial eigenvalues $\lambda$ satisfy the equations

$$
\begin{gathered}
\lambda^{4}+2 \lambda^{2} k_{0}^{2}-2 b_{3} R_{s}^{2}+4 R_{s}^{4}=0, \\
\lambda^{2}\left(\lambda^{2}+2 k_{0}^{2}\right)=0 .
\end{gathered}
$$

The former gives the spatial eigenvalues of the amplitude mode; these are as in the real Swift-Hohenberg equation. The latter equation gives the spatial eigenvalues for the phase mode. Evidently there is always a pair of zero spatial eigenvalues, a consequence of the invariance of the CSHE under phase shifts, together with spatial reversibility. In addition, there is a pair of purely imaginary eigenvalues. Neither of these depends on the state or the value of $r$.

The structure of the spatial eigenvalues of the amplitude mode in the complex plane is given in the insets in Fig. 1. Figure 3 shows the regions with different spatial characteristics of the state $R_{0}=0$ and the upper flat state $R_{0}=R_{s}^{+}$in the $\left(b_{3}, r\right)$ parameter plane when $k_{0}=1$.

Spatial stability of the trivial state. For $r<0$, the spatial eigenvalues of $u=0$ form a complex quartet (not shown). At $r=0$ (point $T_{0}$ ), the eigenvalues collide pairwise on the imaginary axis and a Turing bifurcation takes place, after which $(r>0)$ the eigenvalues split but remain on the imaginary axis (case I). At $r=k_{0}^{4}$ (point $P$ ) two of the eigenvalues collide at the origin (primary steady-state instability) and in $r>k_{0}^{4}$ they move onto the real axis (case II).

Spatial stability of the nonzero flat states. The subcritical flat branch created at $r=k_{0}^{4}$ is characterized by a pair of real eigenvalues and a pair of purely imaginary eigenvalues of the amplitude mode. The former vanish at $r=k_{0}^{4}$ (point $P$ )
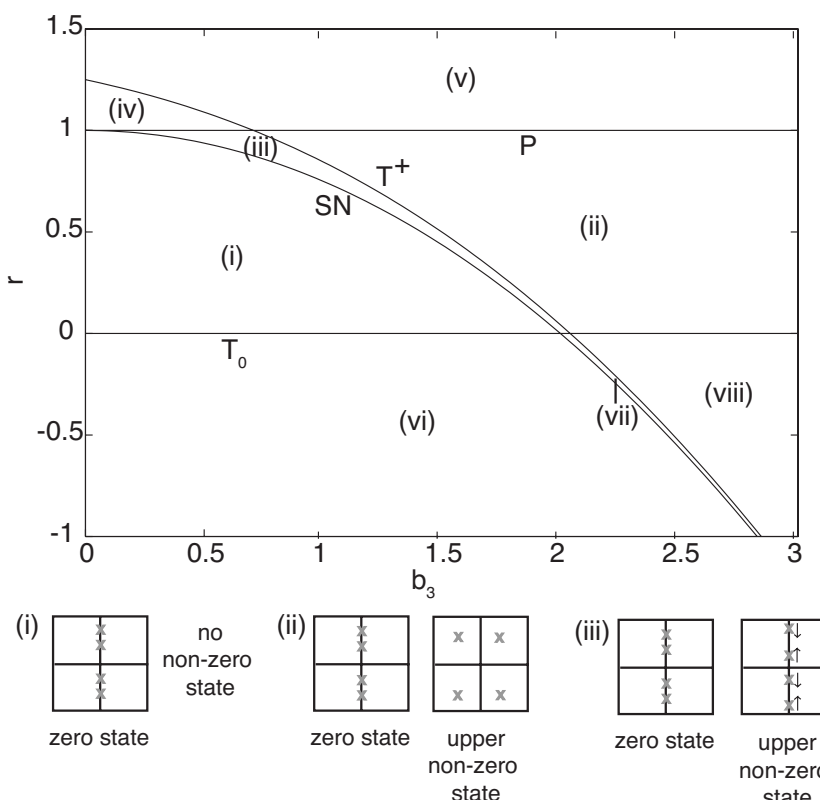

(iii)

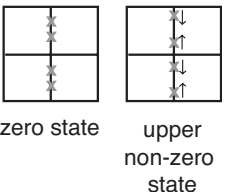

(iv)
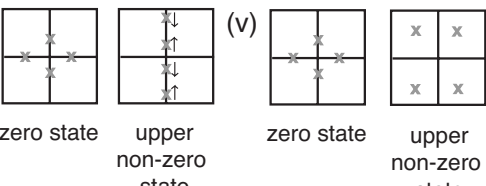

zero state

upper
non-zero
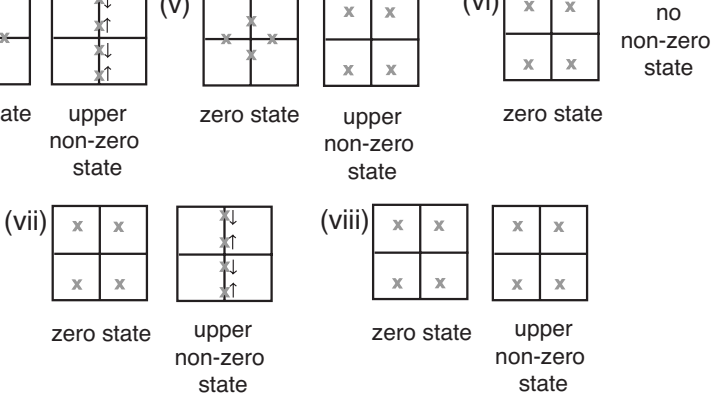

zero state state

state

(viii)
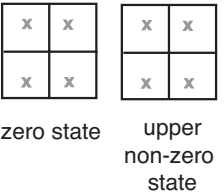

FIG. 3. Structure of the spatial eigenvalues of the trivial $\left(R_{0}\right.$ $=0)$ and upper flat $\left(R_{0}=R_{s}^{+}\right)$states in the $\left(b_{3}, r\right)$ parameter plane when $k_{0}=1$.

and split along the real axis (case III) before returning to zero at $\left(r, u_{s}\right)=\left(-b_{3}^{2} / 4+k_{0}^{4}, \pm \sqrt{b_{3} / 2}\right)$ (point $\mathrm{SN}$ ), where the flat branch folds. After the fold, the pair of zero eigenvalues moves onto the imaginary axis (case IV); at $r=(1 / 8)\left(-b_{3}^{2}\right.$ $+10 k_{0}^{4}-b_{3} \sqrt{b_{3}^{2}+4 k_{0}^{4}}$ ) (point $T^{+}$) these eigenvalues collide pairwise with existing imaginary eigenvalues at $\lambda= \pm i k_{0}$ and split, forming a quartet of complex eigenvalues (case V). This point thus also corresponds to a Turing bifurcation. Spatial reversibility implies that LSs biasymptotic to $u=0$ are structurally stable when $r<0$ [regions (vi)-(viii) of Fig. 3] and likewise for LSs biasymptotic to $u=u_{s}^{+}$in regions (ii), (v), and (viii) of Fig. 3.

\section{Homoclinic snaking of localized states}

Figure 4(a) shows the branch of spatially periodic states emerging at the modulational instability at $r=0$ in terms of its $L^{2}$ norm per unit length (hereafter, energy), together with the (four) classical snaking branches of LSs emerging from this point [11]. We refer to these structures as $\pm u_{\text {even }}$ and $\pm u_{\text {odd }}$ according to their parity: the LSs along the black snaking curve are invariant under $\left(x, \phi_{s}\right) \rightarrow\left(-x, \phi_{s}\right)$; the LSs along the gray snaking curve are invariant under $\left(x, \phi_{s}\right) \rightarrow\left(-x, \phi_{s}+\pi\right)[$ Fig. 4(b)]. At each turn in the snaking 

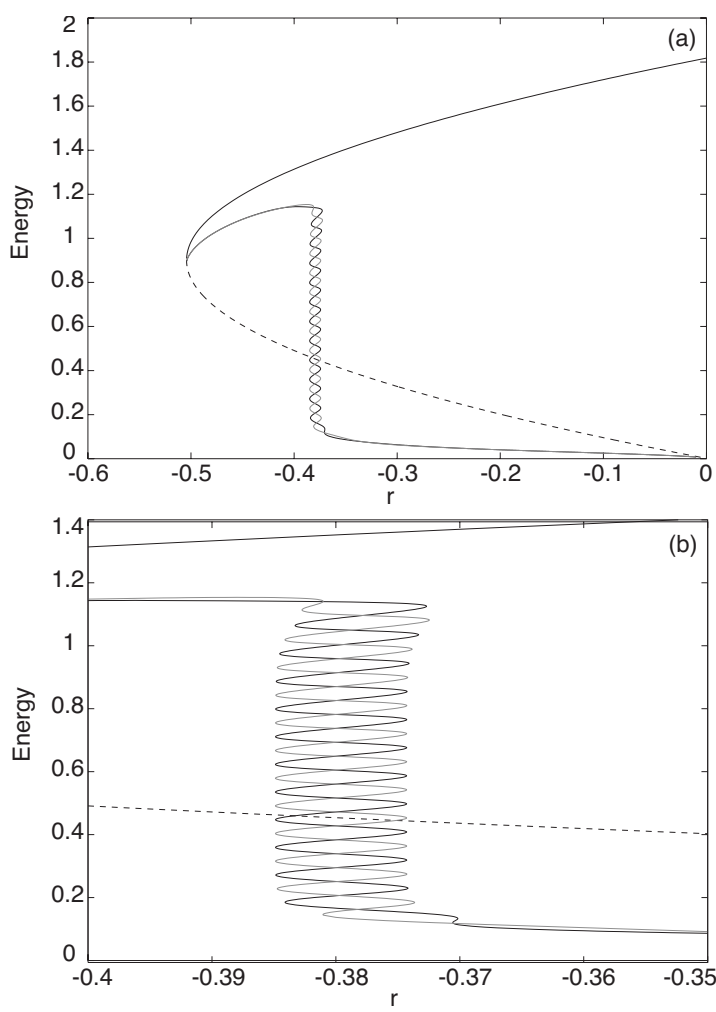

FIG. 4. (a) The periodic pattern SW (dashed line: unstable; solid line: stable) emerging at the modulational instability at $r=0$, together with the classical snaking branches emerging from the same point. The black (gray) snaking branch corresponds to even (odd) parity LS. (b) Zoom of the homoclinic snaking region. Parameters: $b_{3}=1.5, k_{0}=1, L=96$, and $N=512$.

branch an extra half wavelength of the periodic state is added on either side of the LS until the entire domain is filled and the branches exit the snaking region and connect to the periodic pattern branch $[11,26]$. In the following we refer to the periodic pattern as a standing wave (SW) since the real and imaginary parts $\left(u_{R}, u_{I}\right)$ of $u(x)$ oscillate in (spatial) phase: in the $\left(u_{R}, u_{I}\right)$ plane the SW corresponds to an oscillation along an arbitrarily oriented radial line $[27,28]$.

\section{Numerical method}

The numerical time integration method used throughout the paper is a two-step pseudospectral method that calculates the linear part of the equations in Fourier space, while evaluating the nonlinear terms in real space [29,30]. Periodic boundary conditions are used, and the system size $L$ is taken large enough to ensure that the LSs are much smaller than the domain length, thereby avoiding boundary effects. The space discretization is defined by $d x=L / N$ (with $N=512$ or 1024 as the number of discretization points) with a typical temporal time step $d t=10^{-3}$. Stationary profiles found by time integration with appropriate initial conditions are then continued in parameter space using a Newton method [29]. In this method the real and imaginary parts of $u$ in Eq. (2) are discretized in $N$ grid points, resulting in a set of $2 N$ coupled nonlinear equations for the real and imaginary parts of $u$. Although there is no guarantee that all solution branches will
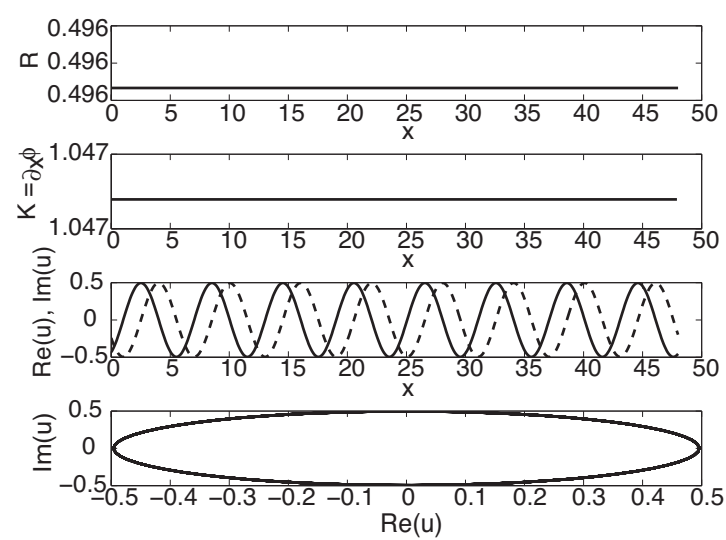

FIG. 5. RW: a phase-winding state in terms of its amplitude $R$ and the modulation wave number $K \equiv \partial_{x} \phi$ (top two panels), the real (solid line) and imaginary (dashed line) parts of the field $u$ (third panel), and the field $u$ in the complex plane (bottom panel). Parameters: $r=-0.3, b_{3}=1.5, k_{0}=1, L=48$, and $N=512$.

be found in this way, an important advantage of the Newton method is that it automatically generates the Jacobian of the system, allowing us to check the stability of the stationary structures as they are computed.

\section{PHASE-WINDING STATES}

In the numerical simulations reported below we encounter states in which the phase is no longer constant in space: over large parts of the domain the phase may vary linearly with the spatial coordinate $x$. We will refer to solutions of this type as phase winding, by analogy with the theory of convection in bounded domains [31], or the description of the director orientation relative to the velocity field in freely suspended liquid crystal films [32].

We first examine how the observed phase gradient is selected and then discuss the temporal and spatial properties of stationary states of the form $u=R_{s} \exp i \phi_{s}, R_{s} \neq 0$, where

$$
R_{s}^{2}=\frac{b_{3} \pm \sqrt{b_{3}^{2}+4\left[r-\left(K^{2}-k_{0}^{2}\right)^{2}\right]}}{2},
$$

$$
\phi_{s}=K x \text {, }
$$

and $K$ is a real constant. In the following we refer to the quantity $K \equiv \partial_{x} \phi$ as the modulation wave number. Figure 5 shows an example of such a state when $r=-0.3, b_{3}=1.5$, and $k_{0}=1$, obtained using periodic boundary conditions at $x$ $=0, L$. Observe that this state is not symmetric under spatial reflection; generically states of this type are expected to drift but here these states are necessarily stationary. This is a consequence of the variational nature of Eq. (2) when the coefficients are real. In view of the constant phase difference (in space) between the real and imaginary parts of the order parameter $u$ [Fig. 5(c)] we refer to phase-winding states of this type as rotating waves $(\mathrm{RWs})$ : in the $\left(u_{R}, u_{I}\right)$ plane RWs correspond to closed orbits around the origin [Fig. 5(d)].

In a phase-winding solution the net phase jump $\Delta \phi$ across a domain of length $L$ must be $2 \pi n$, where $n$ is an integer, in 
order that the phase satisfies the imposed periodic boundary conditions. When this phase jump is uniformly distributed it produces a constant phase gradient specified by $K_{n}$ $\equiv 2 \pi n / L$. Figure 6 shows the branches corresponding to $n$ $=4, \ldots, 10$. The branches with $n=7$ and $n=8$ exist over the widest parameter range and are the "most" stable. In numerical simulations with random initial conditions these are the states that typically result. In the event that $K_{n}=k_{0}$ the corresponding phase-winding solution bifurcates at $r=0$, i.e., si- multaneously with the SW branch shown in Fig. 4. However, although these two periodic states have, in this case, the same wave number $k_{0}$, they are distinct.

\section{A. Temporal stability}

Writing $R=R_{s}+\delta_{R} e^{i k x+\sigma t}$ and $\phi=\phi_{s}+\delta_{\phi} e^{i k x+\sigma t}$, where $R_{s}$ and $\phi_{s}$ are given by Eqs. (17) and (18), one finds that the perturbations $\delta_{R}, \delta_{\phi}$ evolve according to

$$
\left[\begin{array}{cc}
-\left(k^{2}+K^{2}-k_{0}^{2}\right)^{2}-4 K^{2} k^{2}+r+3 b_{3} R_{s}^{2}-5 R_{s}^{4}-\sigma & 4 K i k R_{s}\left(k_{0}^{2}-k^{2}-K^{2}\right) \\
-4 K i k\left(k_{0}^{2}-k^{2}-K^{2}\right) / R_{s} & -k^{2}\left(6 K^{2}-2 k_{0}^{2}+k^{2}\right)-\sigma
\end{array}\right]\left[\begin{array}{l}
\delta_{R} \\
\delta_{\phi}
\end{array}\right]=0
$$

Thus, the temporal growth rate $\sigma$ satisfies the quadratic equation

$$
\begin{aligned}
\sigma^{2}- & \sigma\left[-2 k^{4}-11 K^{2} k^{2}+3 k_{0}^{2} k^{2}+2 b_{3} R_{s}^{2}-4 R_{s}^{4}\right]-k^{2}\left[6 K^{2}-2 k_{0}^{2}+k^{2}\right]\left[-k^{4}+2\left(k_{0}^{2}-K^{2}\right) k^{2}+2 b_{3} R_{s}^{2}-4 R_{s}^{4}-4 K^{2} k^{2}\right]-16 K^{2} k^{2}\left[k_{0}^{2}\right. \\
& \left.-k^{2}-K^{2}\right]^{2}=0 .
\end{aligned}
$$

When $K=0$ this equation reduces to Eqs. (13) and (14). We call the two roots of this equation $\sigma_{1,2}$; Fig. 7(a) shows the larger root $\sigma_{1}$ as a function of $k^{2}$ when $n=10$. The solution is unstable with respect to long-wavelength perturbations above the saddle node (cf. [33]) but stabilizes with increasing $r$ (cf. Fig. 6).

\section{B. Spatial stability}

Writing $R=R_{s}+\delta_{R} e^{\lambda x}$ and $\phi=\phi_{s}+\delta_{\phi} e^{\lambda x}$, where $R_{s}$ and $\phi_{s}$ are given by Eqs. (17) and (18), one finds that the perturbations $\delta_{R}, \delta_{\phi}$ evolve according to

$$
\left[\begin{array}{cc}
-\left(\lambda^{2}+k_{0}^{2}-K^{2}\right)^{2}+4 K^{2} \lambda^{2}+r+3 b_{3} R_{s}^{2}-5 R_{s}^{4} & 4 K \lambda R_{s}\left(k_{0}^{2}+\lambda^{2}-K^{2}\right) \\
-4 K \lambda\left(k_{0}^{2}+\lambda^{2}-K^{2}\right) / R_{s} & \lambda^{2}\left(-2 k_{0}^{2}-\lambda^{2}+6 K^{2}\right)
\end{array}\right]\left[\begin{array}{c}
\delta_{R} \\
\delta_{\phi}
\end{array}\right]=0 .
$$

Thus, the spatial eigenvalues $\lambda$ satisfy

$$
\lambda^{2}\left[\left(\lambda^{4}+2\left(k_{0}^{2}-K^{2}\right) \lambda^{2}-2 b_{3} R_{s}^{2}+4 R_{s}^{4}-4 K^{2} \lambda^{2}\right]\left[2 k_{0}^{2}+\lambda^{2}-6 K^{2}\right]+16 \lambda^{2} K^{2}\left[k_{0}^{2}+\lambda^{2}-K^{2}\right]^{2}=0 .\right.
$$

Once again this equation reduces to Eqs. (15) and (16) when $K=0$. When $K \neq 0$ the pair of zero eigenvalues remains but the remaining sixth-order characteristic equation no longer

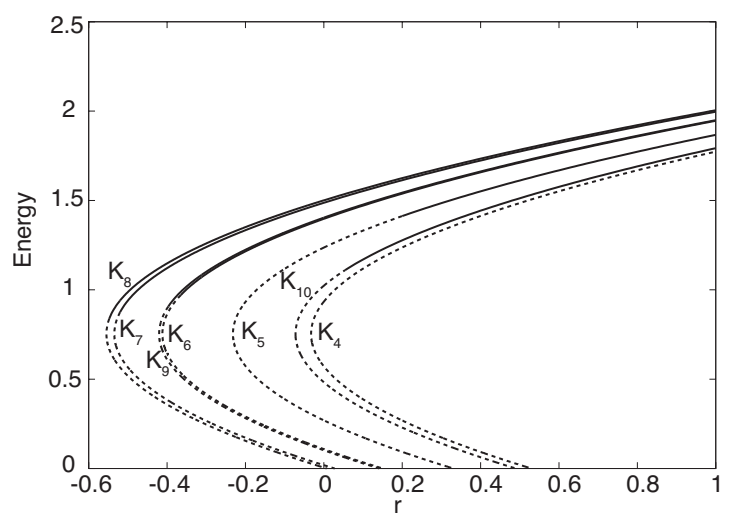

FIG. 6. Seven branches of an infinite family of phase-winding states with constant amplitude and linearly varying phase $\phi=K_{n} x$, where $K_{n} \equiv 2 \pi n / L(n=1,2, \ldots)$. Solid (dashed) lines depict stable (unstable) solutions. Parameters: $b_{3}=1.5, k_{0}=1$, and $L=48$. factors. Figure 7(b) shows the $n=10$ phase-winding branch together with insets showing the structure of the spatial eigenvalues as given by Eq. (20). In particular, the saddle node on the branch of phase-winding solutions no longer corresponds to a bifurcation in space.

\section{FACETING OF PHASE-WINDING STATES}

Simulations of the complex Swift-Hohenberg equation [Eq. (2)] typically reveal the presence of LSs that are localized in both amplitude and phase. The observed structures are generally symmetric under reflection in $x$ with pronounced localization in amplitude but extended phase variation that is almost linear, with slope $K$, say, on one side of the symmetry axis and slope $-K$ on the other side. When periodic boundary conditions are imposed on both $R(x)$ and $\phi(x)$, states of this type come in pairs. The resulting phase variation thus resembles a faceted surface; the relatively abrupt changes in the phase gradient are associated with amplitude defects and the widths of these two transition regions are comparable. 

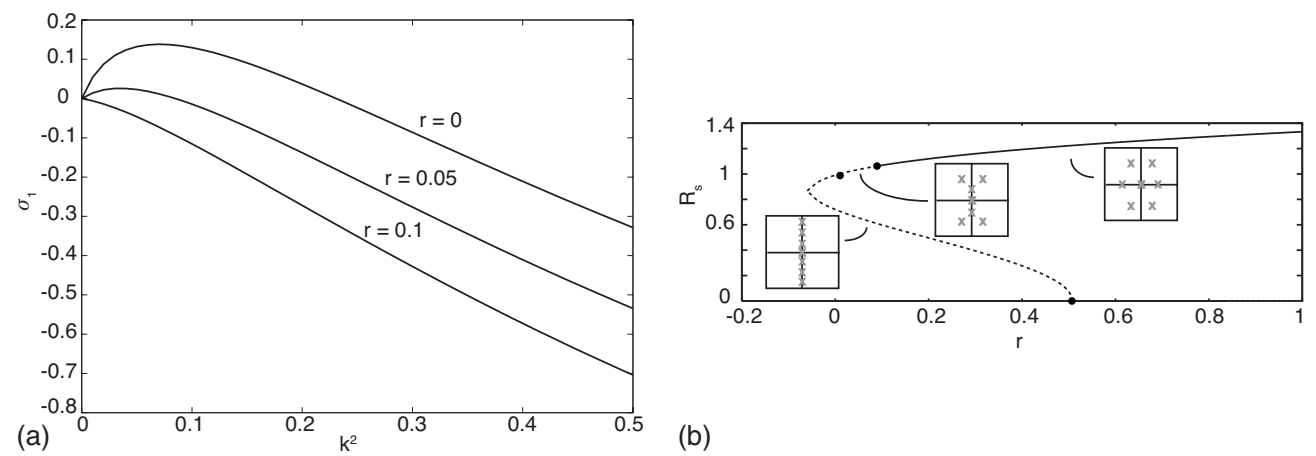

(b)

FIG. 7. (a) Temporal growth rate $\sigma_{1}$ of the $n=10$ phase-winding solution for several values of $r$ when $b_{3}=1.5, k_{0}=1$, and $K_{10}=1.309$ as given by Eq. (19); $\sigma_{2}$ is not shown since it is always more stable. (b) The $n=10$ branch of the phase-winding solutions at the same parameters. The insets show the structure of the spatial eigenvalues of this state as given by Eq. (20). Solid (dashed) lines depict temporally stable (unstable) solutions.

Figure 8(a) shows the branch of in-phase oscillations with wave number $k_{0}$ (SW: labeled II and shown in Fig. 9) that bifurcate from $u=0$ at $r=0$ together with the (four) snaking branches (labeled I) of real-valued LSs that bifurcate from the same point (Fig. 4). Figure 8(a) also shows the first of the branches of the phase-winding states from Eqs. (17) and (18) (see Fig. 6), corresponding to $n=8$ (dark gray curve, labeled III in the figure), found by integrating the complex SwiftHohenberg equation in time, starting from a suitable initial
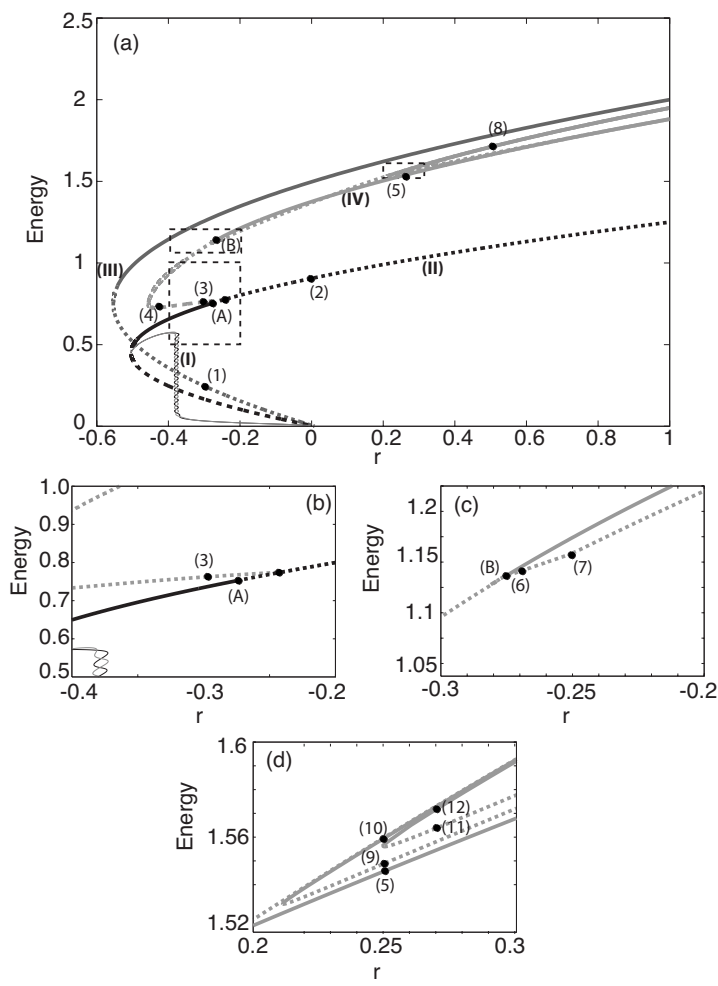

FIG. 8. (a) Bifurcation diagram showing the classical localized (I) and periodic states (II, black line), together with the new phasewinding (III, dark gray line) and faceted (IV, light gray line) states. (b)-(d) depict zooms of the black dashed boxes in (a). The integers indicate locations used for solution profiles in subsequent figures. Solid (dashed) lines depict stable (unstable) solutions. Parameters: $b_{3}=1.5, k_{0}=1, L=48$, and $N=512$. condition. In general, this procedure produces several LSs that are localized in both amplitude and phase [light gray curve, labeled IV in the figure] but in some cases one obtains a constant amplitude phase-winding state. Curve III is obtained from such a state by numerical continuation in the $u_{R}, u_{I}$ variables. Figure 5 shows a sample profile from this branch at location (1). The phase gradient on this branch is constant and essentially independent of the amplitude. As explained in the previous section, this is a consequence of the fact that the phase jump $\Delta \phi$ across a domain of length $L$ must be $2 \pi n$, where $n$ is an integer, in order that the phase satisfies periodic boundary conditions. When the phase varies linearly in space, this phase jump produces the phase gradient $K_{n}=2 \pi n / L$. For branch III of constant amplitude phase-winding state with $n=8$ (Fig. 5), the phase gradient is therefore $K_{8} \approx 1.05$, which is very close to the assumed value of $k_{0}$.

In contrast, the light gray curve, labeled IV, corresponds to continuation from a pair of states that are localized in amplitude and phase. These types of LSs form typically (but not exclusively) when the initial conditions are such that $\Delta \phi=0$. As a consequence we now find a bound state of two
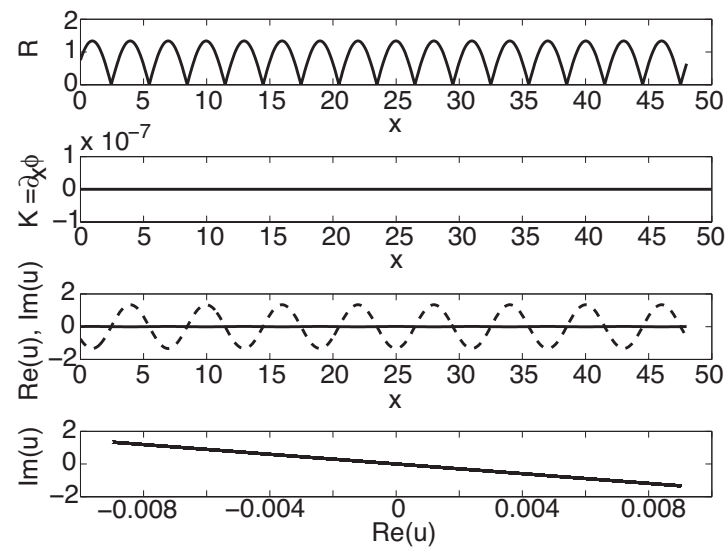

FIG. 9. SW: solution profile at location (2) in Fig. 8(a). Top two panels: amplitude $R$ and modulation wave number $K \equiv \partial_{x} \phi$. Third panel: real (solid line) and imaginary (dashed line) parts of the field $u$. Bottom panel: the field $u$ in the complex plane. Parameters: $r=0, b_{3}=1.5, k_{0}=1, L=48$, and $N=512$. 

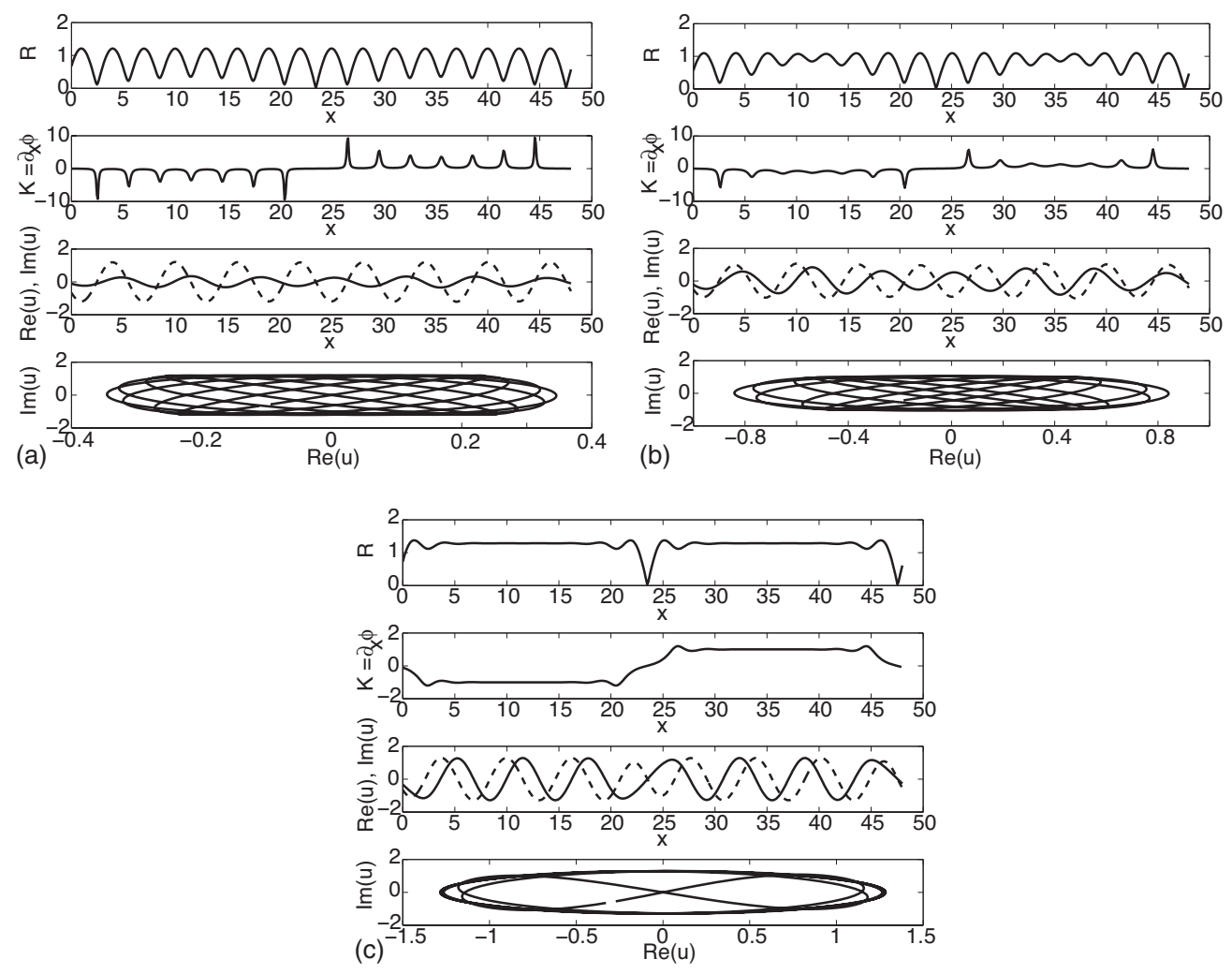

FIG. 10. MW: solution profiles at locations (3), (4), and (5) ( $r=-0.3,-0.45$, and 0.25, respectively) in Fig. 8. Top two panels: amplitude $R$ and modulation wave number $K \equiv \partial_{x} \phi$. Third panel: real (solid line) and imaginary (dashed line) parts of the field $u$. Bottom panel: the field $u$ in the complex plane. Parameters: $b_{3}=1.5, k_{0}=1, L=48$, and $N=512$.

equidistant defects, with opposite chirality. The phase outside these defects varies almost linearly, with alternating gradients $\pm K$. However, in the presence of defects these gradients are no longer selected by the boundary conditions, as is the case for the spatially homogeneous phase-winding states discussed in the previous section. The defects allow the phase gradient $\pm K$ to have the preferred value $\pm k_{0}$ while still satisfying the phase condition $\Delta \phi=0$. The presence of the defects is also reflected in the amplitude $R(x)$ of the state; the profiles of $R(x)$, like those of $K(x) \equiv \partial_{x} \phi$, are symmetric under reflection in $x$. Figure 8(a) shows that as $r$ decreases branch IV of these defect states undergoes a saddle-node bifurcation, while profiles (3)-(5) of Fig. 10 show that below the saddle node the phase gradient becomes markedly nonuniform in space. Moreover, Fig. 8(b) shows that these states connect to the branch of in-phase spatially periodic states (SW) just beyond $A$. This point $A$, at $r=-0.274$, represents a steady-state bifurcation of the SW. The LSs bifurcate from the SW at slightly larger values of $r$, much as the RWs bifurcate from $u=0$ beyond $r=0$, since the solutions are required to be periodic in space with the imposed spatial period $L$ subject to any imposed phase jump $\Delta \phi$. For example, the branch shown in Figs. 8(a) and 8(b) bifurcates from the SW at $r=-0.241$. In space these bifurcations correspond to the spatial analog of the direction-reversing Hopf bifurcation from a group orbit of periodic states discussed in [34]. The resulting LS correspond to (say) a clockwise rotating wave connected to its symmetric partner by a defect. When states of this type are followed toward larger values of $r$, one ob- serves a gradual decrease in nonuniformity in both amplitude and the phase gradient [Fig. 10, profile (5)], and the solution resembles more and more a front connecting equal and opposite wave numbers $\pm K \approx \pm k_{0}$. In the following we refer to states of this type as modulated rotating waves (MWs).

Figure 8(a) also shows that the branch of localized phasewinding states (MW) undergoes a further bifurcation at point $B$ above the saddle node. The new branch of solutions still consists of states with two defects but the two defects are no longer equally spaced [Fig. 11, profiles (6)-(8)]. Details of the region where this bifurcation takes place are shown in Fig. 8(c). We refer to solutions of this type as bound states (BSs).

At larger values of $r$, Figs. 8(a) and 8(d) reveal the presence of several disconnected branches. These branches also correspond to bound states of two identical localized structures. Examples are shown in Figs. 12 and 13. These BSs form via locking between the oscillatory tails of individual localized structures and differ most in the amplitude $R(x)$ of the defects that are involved. Consequently they are part of a whole family of similar BSs with different separations, all on individual branches with similar structure. This behavior is reminiscent of the stacks of disconnected branches extending to arbitrarily large values of the control parameter $r$ that are present in the real Swift-Hohenberg equation with $f_{23}$ nonlinearity in a certain range of values of the parameter $b_{2}$ [7]. In some parameter regimes these change into stacks of isolas. It is possible, therefore, that similar stacks of isolas of defects in the phase-winding states may also exist in the 

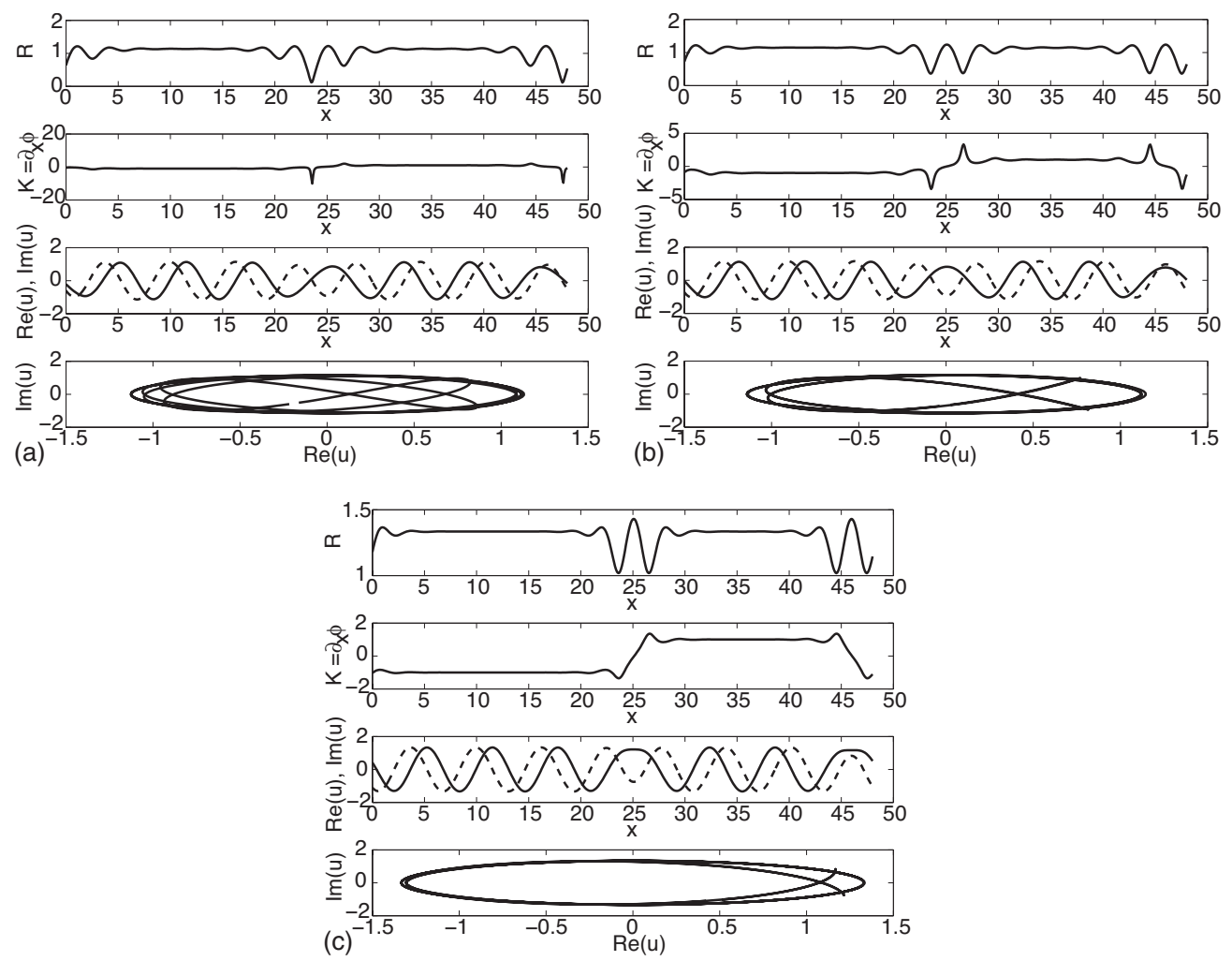

FIG. 11. BS: solution profiles at locations (6), (7), and (8) $(r=-0.27,-0.25$, and 0.5 , respectively) in Fig. 8. Top two panels: amplitude $R$ and modulation wave number $K \equiv \partial_{x} \phi$. Third panel: real (solid line) and imaginary (dashed line) parts of the field $u$. Bottom panel: the field $u$ in the complex plane. Parameters: $b_{3}=1.5, k_{0}=1, L=48$, and $N=512$.

present system. Moreover, bound states of two localized structures in the real Swift-Hohenberg equation lie generically on nested isolas, as described in [35]; the sequence of branches of such states revealed in Figs. 8(a) and 8(d) resembles the structure of one of a stack of such isolas that in the present problem appear to open out to arbitrarily large values of $r$.

\section{COARSENING DYNAMICS: THE CAHN-HILLIARD EQUATION}

In the previous sections we have discussed the presence of LSs, localized both in amplitude and phase. These LSs
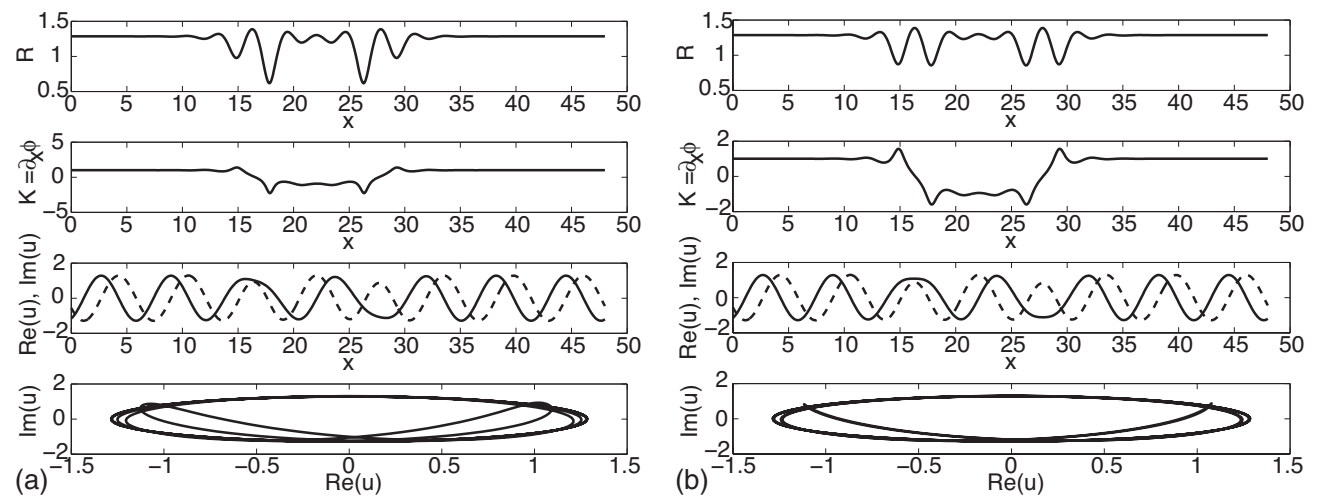

FIG. 12. BS: solution profiles at locations (9) and (10) in Fig. 8. Top two panels: amplitude $R$ and the modulation wave number $K$ $\equiv \partial_{x} \phi$. Third panel: real (solid line) and imaginary (dashed line) parts of the field $u$. Bottom panel: the field $u$ in the complex plane. Parameters: $r=0.25, b_{3}=1.5, k_{0}=1, L=48$, and $N=512$. When rotated by $\pi / 2$ in the complex plane these solutions resemble those in Fig. 11 . have been demonstrated to be associated with phase-winding states in which the real and imaginary parts oscillate out of phase but the phase jumps by an integer multiple of $2 \pi$ across the domain. The LSs take the form of defects connecting phase-winding states with equal and opposite modulation wave numbers $\pm k_{0}$ and hence can be viewed as kinks or antikinks connecting states with opposite phase gradients. As shown in Sec. III, the spatial eigenvalues in the region where these LSs are observed are generally complex, implying that the LSs can be viewed as being the result of locking of the oscillatory tails of each phase. In the present case this Pomeau-type locking [12] leads to a large pinning region in which the LSs are observed. 

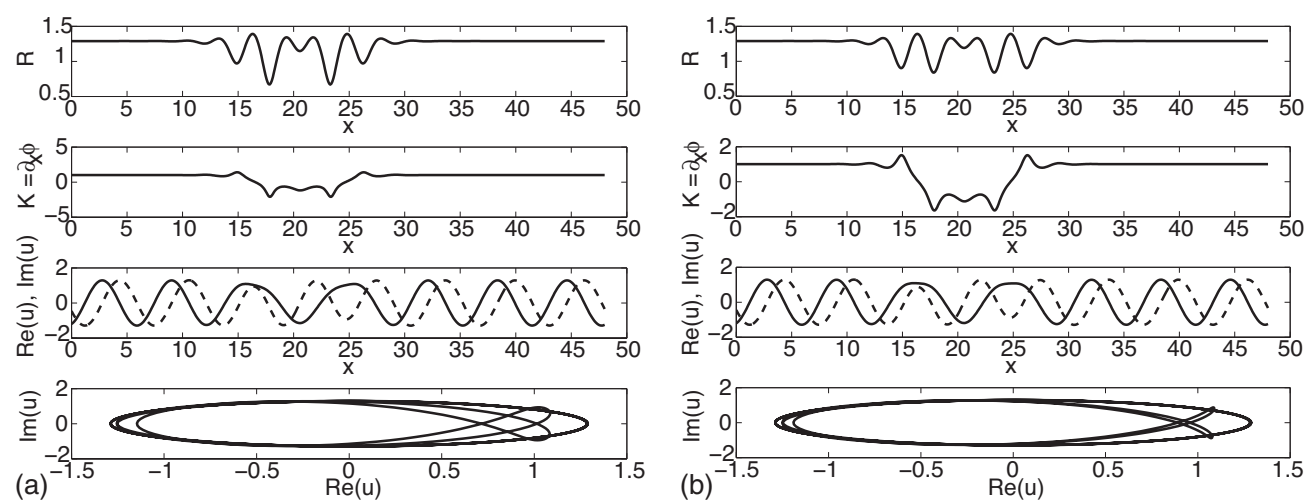

FIG. 13. BS: solution profiles at locations (11) and (12) in Fig. 8. Top two panels: amplitude $R$ and the modulation wave number $K$ $\equiv \partial_{x} \phi$. Third panel: real (solid line) and imaginary (dashed line) parts of the field $u$. Bottom panel: the field $u$ in the complex plane. Parameters: $r=0.27, b_{3}=1.5, k_{0}=1, L=48$, and $N=512$.

In this section, we study the stability of the flat constantphase states with respect to long-wavelength perturbations. The analysis is motivated by the temporal stability results that indicate the presence of a slowly growing longwavelength phase mode [Eq. (14)]. We show that the evolution of long-wavelength perturbations is described by a Cahn-Hilliard-type equation. In the region of validity of this equation, there is no locking of oscillatory tails and coarsening of the growing perturbations is predicted. This prediction compares well with direct numerical simulations of the complex Swift-Hohenberg equation. Extensions of the theory to other regimes do predict locking of adjacent structures and hence evolution to a frozen asymptotic state.

\section{A. Derivation of the Cahn-Hilliard equation}

We consider the evolution of long-wavelength phase modulation of the upper flat state $R_{0}=R_{s}^{+}, \phi_{0}=\phi_{s}$. We first rewrite Eqs. (4) and (5) as coupled equations for the amplitude $R(x, t)$ and modulation wave number $K(x, t) \equiv \partial_{x} \phi(x, t)$ as follows:

$$
\begin{aligned}
R_{t}= & r R+b_{3} R^{3}-R^{5}-2 k_{0}^{2} R_{x x}+6 K^{2} R_{x x}+12 R_{x} K K_{x}-k_{0}^{4} R \\
& -K^{4} R+2 k_{0}^{2} K^{2} R+3 R K_{x}^{2}+4 R K K_{x x}-R_{x x x x} \\
K_{t}= & -2 k_{0}^{2}\left(2 \frac{R_{x}}{R} K+K_{x}\right)_{x}-\left(4 \frac{R_{x x x}}{R} K+6 \frac{R_{x x}}{R} K_{x}+4 \frac{R_{x}}{R} K_{x x}\right. \\
& \left.+K_{x x x}-4 \frac{R_{x}}{R} K^{3}-6 K^{2} K_{x}\right)_{x}
\end{aligned}
$$

Writing $R=R_{0}+u$ with $R_{0}$ a homogeneous solution and $K$ $=v$, where $u=O\left(\epsilon^{2}\right), v=O(\epsilon)$, and $\epsilon \ll 1$ is a small parameter measuring the wave number of the perturbation, one obtains the following equation for $u$ :

$$
\begin{aligned}
u_{t}= & \left(r-k_{0}^{4}+3 b_{3} R_{0}^{2}-5 R_{0}^{4}\right) u+\left(3 b_{3} R_{0}-10 R_{0}^{3}\right) u^{2}+2 k_{0}^{2} R_{0} v^{2} \\
& +2 k_{0}^{2} u v^{2}+3 R_{0} v_{x}^{2}+4 R_{0} v v_{x x}-R_{0} v^{4}-2 k_{0}^{2} u_{x x} \\
& + \text { higher order terms. }
\end{aligned}
$$

We now write $u=\alpha v^{2}+w$, where $\alpha=O(1)$ and $w$ is of order $O\left(\epsilon^{4}\right)$, and take $\partial_{t} \equiv O\left(\epsilon^{4}\right)$, obtaining at leading order (cf. [36])

$$
\begin{gathered}
\alpha=-\frac{2 k_{0}^{2} R_{0}}{r-k_{0}^{4}+3 b_{3} R_{0}^{2}-5 R_{0}^{4}}, \\
w=-\frac{\left[\left(3 b_{3}-10 R_{0}^{2}\right) R_{0} \alpha^{2}+2 k_{0}^{2} \alpha-R_{0}\right] v^{4}}{r-k_{0}^{4}+3 b_{3} R_{0}^{2}-5 R_{0}^{4}} \\
-\frac{\left[3 R_{0}-4 k_{0}^{2} \alpha\right] v_{x}^{2}+4\left[R_{0}-k_{0}^{2} \alpha\right] v v_{x x}}{r-k_{0}^{4}+3 b_{3} R_{0}^{2}-5 R_{0}^{4}} .
\end{gathered}
$$

With $u=\alpha v^{2}+w$, Eq. (22) yields to $O\left(\boldsymbol{\epsilon}^{7}\right)$ the result

$$
\begin{aligned}
v_{t}= & {\left[-2 k_{0}^{2} v-v_{x x}-\kappa_{0} v^{3}-\kappa_{1} v^{5}\right]_{x x} } \\
& -c\left(v^{2} v_{x x x}\right)_{x}-d\left(v v_{x} v_{x x}\right)_{x}-e\left(v_{x}^{3}\right)_{x}+\text { higher order terms. }
\end{aligned}
$$

Expressions for the coefficients in this equation can be found in the Appendix.

The above derivation is asymptotically consistent provided we take $k_{0}=O(\epsilon)$ in which case we obtain at leading order the Cahn-Hilliard equation [37]

$$
v_{t}=\left[-2 k_{0}^{2} v-v_{x x}-\kappa_{0} v^{3}\right]_{x x}
$$

with

$$
\kappa_{0}=-2\left[1+\frac{8 k_{0}^{4}}{3\left(r-k_{0}^{4}+3 b_{3} R_{0}^{2}-5 R_{0}^{4}\right)}\right] \approx-2 .
$$

Alternatively we can take $k_{0}$ to be larger, $k_{0}=o(\epsilon)$, take $v$ $=o(\epsilon)$, and rescale length and time to obtain Eq. (27) in its more usual form with a small coefficient in front of $v_{x x}$. Such a rescaling is possible without reintroducing any of the higher order terms in Eq. (27).

The Cahn-Hilliard equation was originally derived to describe the dynamics of phase separation in systems with a conserved quantity in the context of binary alloys [37]. However, the model equation arises in many other areas of physics as well [38], including spinodal decomposition in thin films [39], pattern formation on surfaces, dislocations of microstructures, crack propagation, and electromigration, where it is used to describe progressive coarsening [38]. In the present context the form of the equation is a consequence of phase conservation across the domain, together with the 


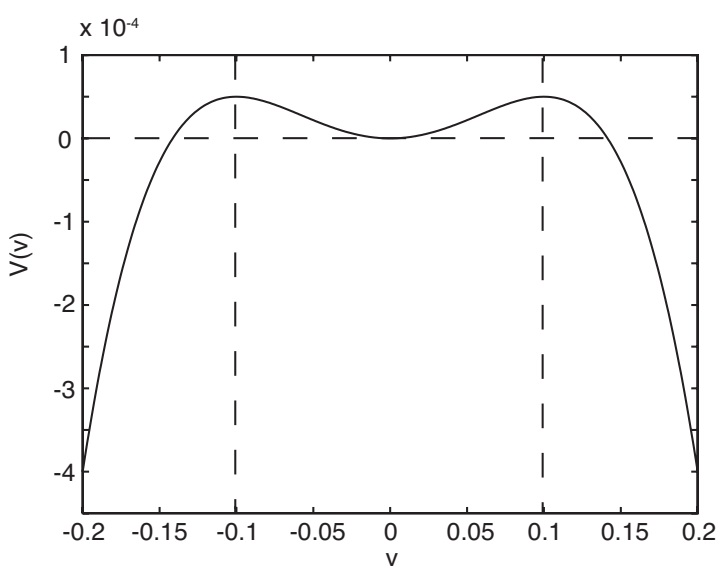

FIG. 14. Potential $V(v)$ as a function of $v$ for $k_{0}=0.1$.

symmetry $R \rightarrow R, K \rightarrow-K$ under spatial reflection present in Eqs. (21) and (22). In the following we seek solutions of the Cahn-Hilliard equation (27) with zero mean, so that $\Delta \phi=0$ across the domain, as required of any perturbation of a constant-phase state.

\section{B. Stationary solutions of the Cahn-Hilliard equation}

The Cahn-Hilliard equation, Eq. (27), has the Lyapunov functional

$$
F(v)=\int d x\left[\frac{1}{2} v_{x}^{2}-V(v)\right],
$$

where (see Fig. 14)

$$
V(v) \equiv k_{0}^{2} v^{2}+\frac{1}{4} \kappa_{0} v^{4},
$$

defined such that

$$
v_{t}=\partial_{x x}[\delta F(v) / \delta v(x)] .
$$

The stationary states are easily found by integrating the stationary equation twice,

$$
v_{x x}+2 k_{0}^{2} v+\kappa_{0} v^{3}+\lambda_{1}+\lambda_{2} x=0,
$$

where $\lambda_{1}, \lambda_{2}$ are integration constants. Symmetry with respect to reflection in $v$ requires that $\lambda_{1}=0$, while reflection in $x$ implies that $\lambda_{2}=0$. Thus,

$$
\frac{1}{2} v_{x}^{2}+V(v)=E \text {. }
$$

We refer to the integration constant $E$ as the energy.

Since $V$ has two identical maxima $\left(\kappa_{0} \approx-2\right)$, there is a pair of symmetry-related spatially homogeneous steady states given by

$$
v_{s} \approx \pm k_{0}
$$

with energy $E_{s}=-k_{0}^{4} / \kappa_{0}>0$. When $0<E<E_{s}$, there is a family of spatially periodic nonlinear solutions of zero mean whose period and amplitude depend on $E$. These solutions are, however, known to be unstable [40,41]. As $E \rightarrow E_{s}$ from below these solutions degenerate into a pair of heteroclinic states connecting a pair of symmetric equilibria. These states are called kinks if the phase increases across the associated
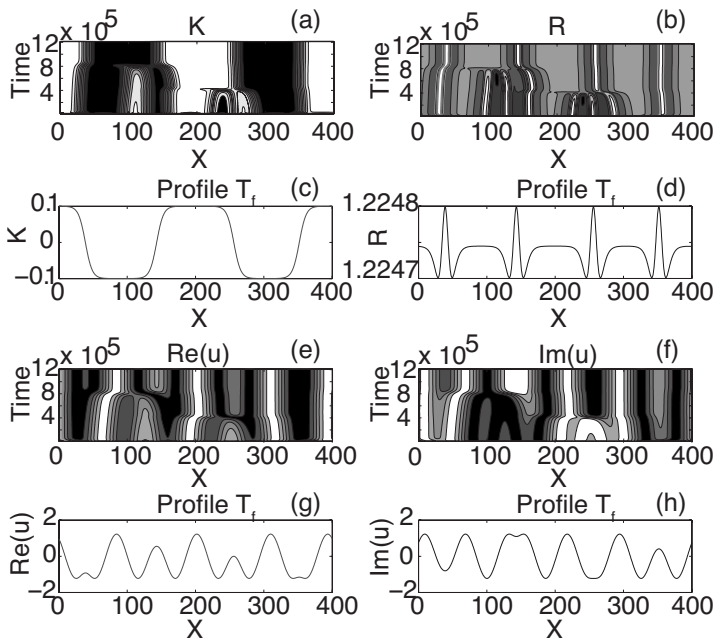

FIG. 15. Spatiotemporal evolution of the CSHE from an initial condition with $n=0$ consisting of four bubbles of different widths. Parameters: $r=0, b_{3}=1.5, k_{0}=0.1, L=400$, and $N=1024$. (a) and (b) Space-time contour plot of $K \equiv \partial_{x} \phi$ and the amplitude $R$. (c) and (d) The final profile of $K$ and $R$ taken from the last time step in (a) and (b). (e) and (f) Space-time contour plot of the real and imaginary parts of the complex field $u$. (g) and (h) The final profile of $\operatorname{Re}(u)$ and $\operatorname{Im}(u)$ taken from the last time step in (e) and (f). In the contour plots high (low) amplitude is color coded by white (black).

defect, and antikinks if it decreases. A kink-antikink pair is the long-time zero-area attractor of the equation [40-42].

The knowledge of these attracting stationary solutions of the Cahn-Hilliard equation allows us to gain a qualitative picture of the spatiotemporal dynamics in the CSHE in the parameter regime in which Eq. (27) applies. Previous work [42-47] has demonstrated that particlelike or defect solutions, localized in space, can exist in the Cahn-Hilliard equation. These solutions are sometimes referred to as bubbles, that is, bound pairs of kink-antikink profiles connecting two homogeneous states (in our case $v_{s} \approx \pm k_{0}$ ). A single bubble is a stable solution of the equation since the evolution of the system conserves the area $A \equiv \int_{0}^{L} v(x, t) d x$. However, a system consisting of several bubbles is unstable and in time exhibits coarsening dynamics, in which smaller bubbles repeatedly merge together forming larger and larger structures. This process is driven by the mutual interaction between the kink and antikink pairs and is present whenever the spatial eigenvalues of $v_{s} \approx \pm k_{0}$ are real, i.e., provided there is no pinning. It is known that at large times the width of the broadest bubble increases exponentially slowly as a result of the exponentially small interaction between kinks and antikinks when these are widely separated $[48,49]$.

\section{Numerical verification of coarsening dynamics}

In the following we demonstrate that the CSHE equation does indeed follow the coarsening dynamics predicted from the Cahn-Hilliard equation and discuss the origin of any differences from Cahn-Hilliard dynamics. In Fig. 15, we show a temporal simulation of the CSHE for $r=0, k_{0}=0.1$, starting from an initial condition consisting of four bubbles of different widths and separations. For these parameters Eq. (27) 
predicts that $K_{s} \approx \pm 0.1$. For these small values of $K_{s}$ we expect the spatiotemporal dynamics of the CSHE to follow the coarsening behavior of the Cahn-Hilliard equation. Figure 15 shows that this is indeed the case. Figures 15(a) and 15(b) show the annihilation of the first two bubbles. The profiles of the phase gradient $K \equiv \partial_{x} \phi$ and the amplitude $R$ at the last time step in Figs. 15(a) and 15(b) are shown in Figs. 15(c) and 15(d), respectively; the panels show that the kinks and antikinks indeed form connections between the states $K_{s}= \pm 0.1$, as expected. Figures $15(\mathrm{e})-15(\mathrm{~h})$ show this process in terms of the real and imaginary parts of the complex field $u$. For longer simulation times, the Cahn-Hilliard equation predicts that the bubbles continue to coarsen until only one bubble remains, whose area (and hence width) is determined by the initial condition. However, since the annihilation of the bubbles becomes exponentially slow as the width and/or separation of the bubbles increases the very slow dynamics that leads to the final state is not shown. Advanced numerical schemes to study this process in one spatial dimension have been developed and these have confirmed the metastable dynamics and very slow coarsening starting from a large number of bubbles in the initial state [50].

Within the Cahn-Hilliard equation the interaction between two or more bubbles has been studied in detail $[42,50]$. The results provide a description of the coarsening process in terms of the motion of the kinks and antikinks defining the bubbles. Since the Cahn-Hilliard equation conserves area, during an interaction between two disparate bubbles the global center of area moves in the direction of the widest bubble, and the widest bubble increases its width and moves slightly toward the thinner one [42]. For comparison, Fig. 16 shows the details of an interaction between two bubbles in the CSHE. The figure shows that in the CSHE the two bubbles do indeed attract each other in such a way that the widest bubble increases its width and moves slowly toward the narrower one. After annihilation of the smaller bubble, the remaining bubble relaxes and grows until it takes up half of the domain. The time evolution of the width of the broadest bubble is depicted in Fig. 16(e). The width $d_{2}$ has been defined as the region of the bubble in which $K<0$. In region A, the widths of the bubbles are still adjusting themselves to the initial condition of the simulation. In region $\mathrm{B}$, however, one can clearly see that the width of the largest bubble increases with time. At time $t^{*}$ the last two bubbles merge, and in region $\mathrm{C}\left(t>t^{*}\right)$ the remaining bubble relaxes exponentially slowly to the global minimum of the functional $F(v)$ in Eq. (29): with periodic boundary conditions this global minimum consists of one bubble filling half the domain. The profile of the stable final bubble solution is shown in more detail in Figs. 16(b) and 16(d) in terms of its phase gradient $K \equiv \partial_{x} \phi$ and the phase $\phi$ itself. For comparison, we show in Fig. 16(c) the phase $\phi$ at an earlier time $t=2 \times 10^{5}$. We conclude that the CSHE does indeed follow the expected CahnHilliard type of coarsening dynamics when $k_{0}$ is sufficiently small that the derivation of the Cahn-Hilliard equation [Eq. (27)] is self-consistent.

We have also performed simulations for the same parameter set, but using initial conditions with a net phase jump $\Delta \phi=2 \pi n$ across the domain, where $n$ is an integer. Since the phase jump is conserved by the evolution such an initial
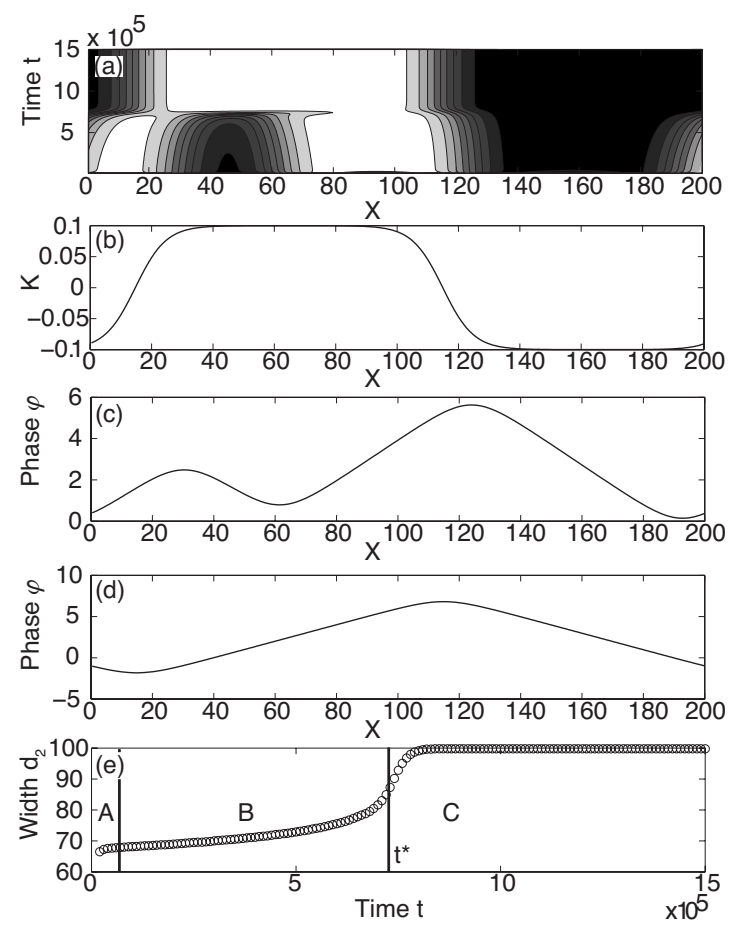

FIG. 16. Spatiotemporal simulation of the CSHE from an initial condition with $n=0$ consisting of two bubbles of different widths. Parameters: $r=0, b_{3}=1.5, k_{0}=0.1, L=200$, and $N=512$. (a) Spacetime contour plot of $K \equiv \partial_{x} \phi$. High (low) $K$ is color coded by white (black). (b) The final profile of $K$ taken from the last time step in (a). (c) and (d) Phase profiles $\phi$ at $t=2 \times 10^{5}$ and at the final time $t=1.5 \times 10^{6}$, respectively. (e) The time evolution of the width $d_{2}$ of the broadest bubble.

condition corresponds to a perturbation around a state with phase gradient $K=2 \pi n / L$, i.e., a phase-winding state, instead of the case $K=0$ for which the Cahn-Hilliard equation has been derived. For this case $\lambda_{1} \neq 0$ in Eq. (32), changing the potential $V(v)$ depicted in Fig. 14. However, if we allow only a phase jump $\Delta \phi$ such that $K=2 \pi n / L$ and $\lambda_{1}$ remain small, the inverted double-well potential changes only slightly and one expects the coarsening process to persist, at least for long times. This is the case in Fig. 17 which shows the evolution of an $n=2$ initial condition on a domain of length $L$ $=300$. The coarsening is now observed for finite times only, although the most prominent difference between the behavior of the CSHE and the Cahn-Hilliard equation is seen in the relaxation to the final one-bubble solution. Here, the imposed phase gradient, small though it is, pins the bubble and prevents it from relaxing to half the domain length. Indeed, we observe almost no relaxation once the two bubbles merge. As a result the size $d_{2}$ of the last bubble changes only slightly as $t \rightarrow \infty$. Throughout the entire time evolution $\Delta \phi=4 \pi=12.57$ and we may use this fact to write down an approximate expression for the final bubble size $d_{2}$,

$$
2 n \pi=v_{s}\left(d_{1}-d_{2}\right) \approx k_{0}\left(L-2 d_{2}\right),
$$

implying that 

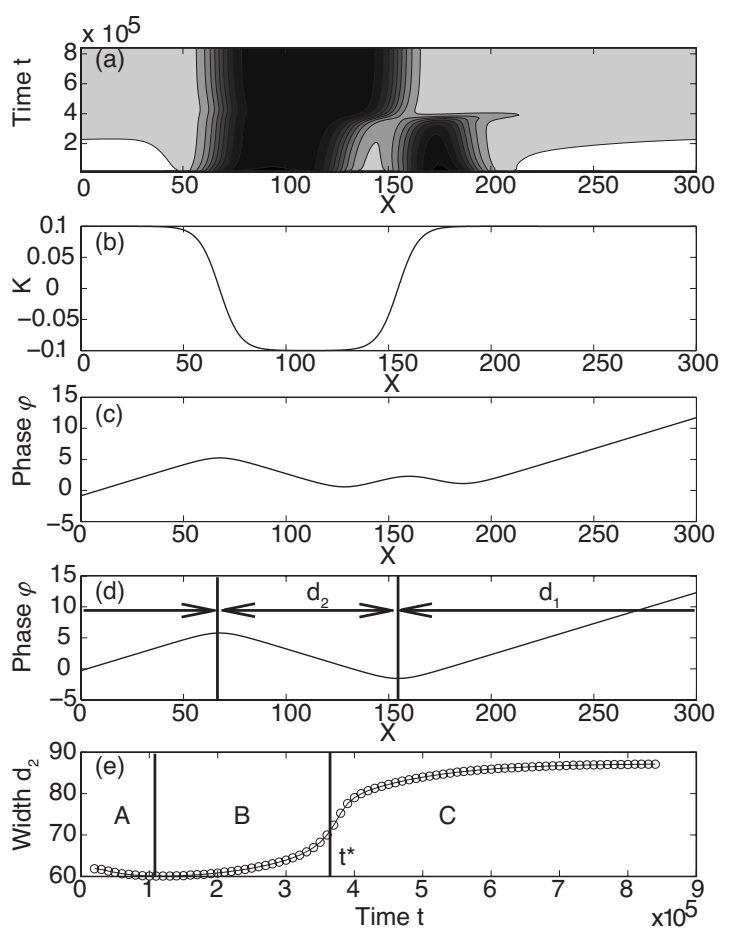

FIG. 17. Spatiotemporal simulation of the CSHE from an initial condition with $n=2$ consisting of two bubbles of different widths. Parameters: $r=0, b_{3}=1.5, k_{0}=0.1, L=300$, and $N=1024$. (a) Spacetime contour plot of $K \equiv \partial_{x} \phi$. High (low) $K$ is color coded by white (black). (b) The final profile of $K$ as taken from the last time step in (a). (c) and (d) Phase profiles $\phi$ at $t=2 \times 10^{5}$ and at the final time $t=8.4 \times 10^{5}$, respectively. (e) The time evolution of the width $d_{2}$ of the broadest bubble.

$$
d_{2}=\frac{L}{2}-\frac{\pi n}{k_{0}},
$$

with $n$ an integer determined by the initial conditions (here $n=2$ ). For the parameters chosen in Fig. 17, Eq. (35) yields $d_{2}=87.2$, which agrees very well with the final bubble size observed in Fig. 17.

\section{Transition from coarsening to faceting behavior}

As discussed in Sec. IV, coarsening behavior is no longer present for larger values of $k_{0}$. Instead one observes faceting in which fronts connecting equal and opposite phase gradients $K$ are pinned to each other, permitting the coexistence of multiple stable bubbles. Direct numerical simulations of the CSHE [Eq. (2)] show that these fronts always connect states with $K= \pm k_{0}$. These simulations thus extend the conclusion obtained analytically from the Cahn-Hilliard equation derived in Sec. V A for small values of $k_{0}$ to much larger values of $k_{0}$.

In order to predict the value of $k_{0}$ at which the transition from coarsening to faceting behavior takes place we examine the spatial eigenvalues of the phase-winding state with $K$ $=k_{0}$ as described by Eq. (20). Since the decay of spatial perturbations around this phase-winding state is controlled by the slowest eigenvalue, i.e., the eigenvalue with the smallest nonzero real part, coarsening will take place whenever this eigenvalue is purely real and oscillatory tails are absent. On the other hand, whenever this particular eigenvalue has a nonzero imaginary part oscillatory tails will be present allowing adjacent kink and antikinks to lock to one another [12]. In this case one expects to observe faceting. It follows, therefore, that the transition between coarsening and faceting dynamics is given by the point in parameter space where the eigenvalue with the smallest real part acquires a nonzero imaginary part.

In Fig. 18 we show the real and imaginary parts of the spatial eigenvalues of a phase-winding state with modulation wave number $K$ in the special case in which $K=k_{0}$. This choice is motivated by our numerical simulations which show that the phase gradients involved in the formation of a localized structure are always $k_{0}$. For the parameters $r=1$, $b_{3}=1.5$, and $K \equiv k_{0} \in[0,1]$, the eight eigenvalues [as obtained from Eq. (20)] are organized as follows: there is a quartet of complex eigenvalues $\left(\lambda_{1,2,3,4}= \pm \lambda_{r} \pm i \lambda_{i}\right)$, two purely real eigenvalues $\left(\lambda_{5}=-\lambda_{6}\right)$ and a double zero eigenvalue $\left(\lambda_{7,8}=0\right)$. As already mentioned zero eigenvalues are a consequence of the invariance of the CSHE under phase shifts, together with spatial reversibility. From Fig. 18, it is clear that there is a crossover between the magnitude of the
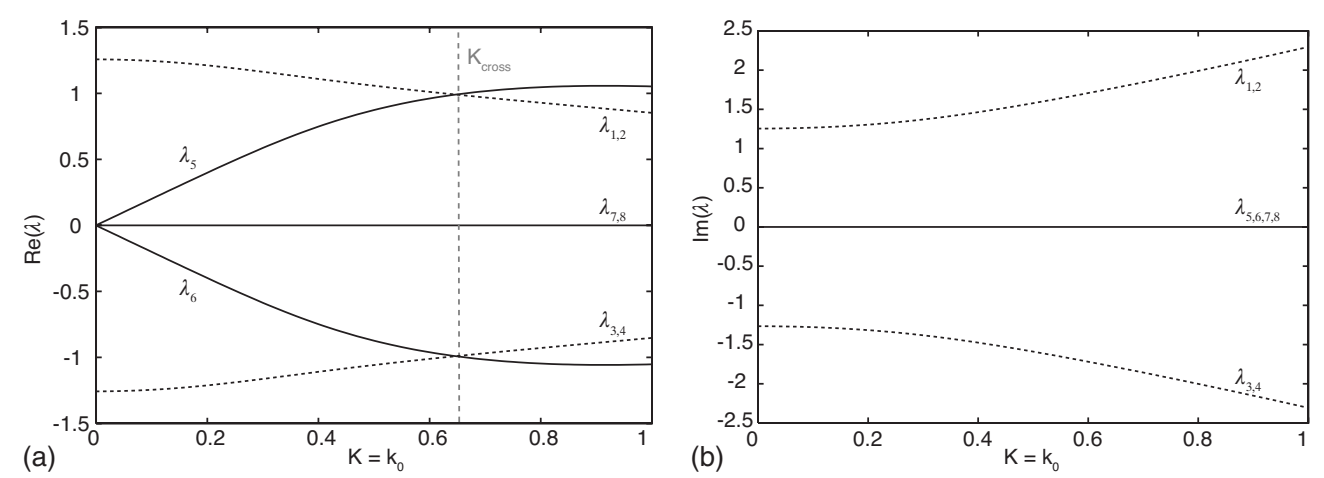

FIG. 18. The (a) real and (b) imaginary parts of the spatial eigenvalues of the phase-winding state as a function of the phase gradient $K$, $0 \leq K \leq 1$, and $K$ set equal to $k_{0}$ as observed in all numerical simulations resulting in localized states. Thus, the plot also represents the variation of the real and imaginary parts of the relevant spatial eigenvalues as a function of the basic wave number $k_{0}$. Parameters: $r=1$ and $b_{3}=1.5$. 

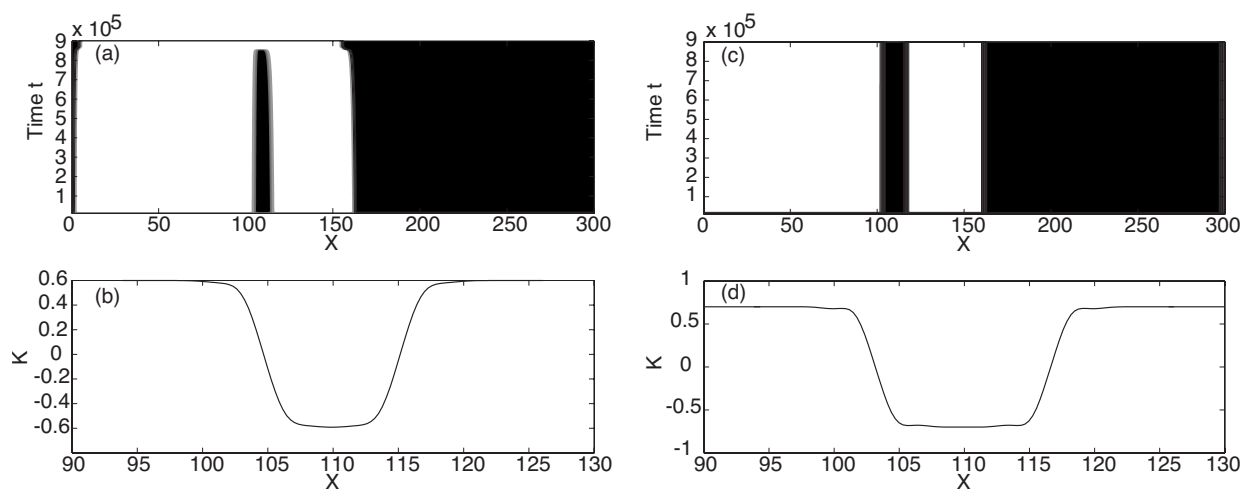

FIG. 19. Spatiotemporal simulation of the CSHE from an initial condition with $n=0$ consisting of two bubbles of different widths. Parameters: $r=1, b_{3}=1.5, L=300, N=1024$, and $k_{0}=0.6$ [0.7] in (a) [(c)] and (b) [(d)], respectively. (a) and (c) show the space-time contour plot of $K \equiv \partial_{x} \phi$. High (low) $K$ is color coded by white (black). (b) and (d) show a zoom of the profiles in $K$ of the small bubbles at $t=5$ $\times 10^{4}$ taken from (a) and (c).

real parts of the complex quartet and the purely real eigenvalues. This crossover occurs around $K_{\text {cross }} \approx 0.65$ and splits the graph in two regions: a coarsening region for $K=k_{0}$ $<K_{\text {cross }}$ and a faceting region where the LS can pin to the oscillatory tails of the fronts $\left(K=k_{0}>K_{\text {cross }}\right)$. This prediction of the structure of the spatial eigenvalues is verified in Fig. 19, where we show two temporal simulations of the CSHE: one for $K=k_{0}=0.6<K_{\text {cross }}$ [Fig. 19(a)] and one for $K=k_{0}$ $=0.7>K_{\text {cross }}[$ Fig. $19(\mathrm{c})]$. In both cases we use the same initial condition consisting of two bubbles - one of which is much smaller in width than the other one-and identical values of $r, b_{3}$, and $L$. The results reveal an unambiguous qualitative change in the dynamical behavior of the system from coarsening to faceting. Moreover, the front profile of the small bubble [Figs. 19(b) and 19(d)] confirms the absence or presence of oscillatory tails when coarsening or faceting takes place.

\section{CONCLUSIONS}

We have described a class of states in the bistable complex Swift-Hohenberg equation with real coefficients, which we have called phase-winding states. These complex-valued solutions oscillate periodically in space, like the periodic states of the real Swift-Hohenberg equation, but with a welldefined phase difference between the real and imaginary parts. The solutions fall into different families characterized by the overall phase jump across the domain, which must be a nonzero integer multiple of $2 \pi$. These states are easily found numerically in the part of parameter space where spatially uniform and in-phase periodic states are unstable, and then followed to other regions of parameter space.

Associated with the spatially extended phase-winding states one finds a class of localized states taking the form of defects connecting phase-winding states with equal and opposite phase lag. The resulting phase defect is reflected in the amplitude of the complex field as well. These types of localized structures appear to be related to the defect states recently described by Burke et al. [51] and Ma et al. [52] in the context of the forced Ginzburg-Landau equation.

The spatially extended phase-winding states and the defect states both exist over a wide range of parameters. The latter are organized in a structure closely resembling that identified in the real Swift-Hohenberg equation with a $f_{23}$ nonlinearity that organizes localized states in a nonzero but homogeneous background [7]. In both cases stacks of disconnected branches that extend to arbitrarily large values of the control parameter $r$ are observed.

These defects correspond to fronts connecting equal and opposite phase gradients. The fronts that are observed for order-one wave number $k_{0}$ have oscillatory tails allowing Pomeau locking between kinks and antikinks, stabilizing the localized states. In this region the phase variation resembles a faceted surface with abrupt changes in the phase gradient. On the other hand, when the characteristic wave number $k_{0}$ is small, stable facets and defects are no longer present. Instead, numerical simulations reveal coarsening dynamics of the fronts. A theoretical analysis of the stability of the zero phase gradient state with respect to long-wavelength perturbations has led to the well-known Cahn-Hilliard equation for the perturbation phase gradient, thereby confirming the coarsening results obtained from simulations of the SwiftHohenberg equation [Eq. (2)].

Our study of the bistable CSHE with real coefficients should prove invaluable for a deeper understanding of the complex coefficient case [53] that describes subcritical oscillatory systems with a finite onset wave number $[15,16]$. Indeed, preliminary results show that similar faceting behavior of phase-winding states occurs in the complex coefficient case as well. Moreover, an examination of our results suggests that similar faceting should also take place in the supercritical case and hence in two-level class $\mathrm{A}$ and $\mathrm{C}$ lasers $[17,18]$, nondegenerate OPOs [20-22], photorefractive oscillators [23], and semiconductor lasers [24] all of which are described by the supercritical CSHE. However, the bistable CSHE with complex coefficients does describe passively mode-locked lasers in appropriate regimes [25] and this system offers therefore the best opportunity for an experimental realization of the behavior described here.

We mention, finally, that one-dimensional fronts in the two-dimensional Swift-Hohenberg equation for a real order parameter may also undergo a faceting or zigzag instability $[54,55]$. This instability results in a faceted front and is distinct from the faceting in the spatial phase of the pattern described here. 


\section{ACKNOWLEDGMENTS}

This work was funded by the Belgian Science Policy Office under Grant No. IAP-VI10. Support from the Research Foundation-Flanders (L.G.) and from the National Science Foundation under Grant No. DMS-0605238 (E.K.) is gratefully acknowledged. We thank J. Burke, S. Houghton, and U. Thiele for helpful discussions and a referee for bringing several references to our attention.

\section{APPENDIX: COEFFICIENTS IN THE CAHN-HILLIARD EQUATION}

As shown in Sec. V, the evolution of small perturbations of the phase gradient $K \equiv \partial_{x} \phi$ around $K=0$ is governed by Eq. (27) obtained as the leading order balance in the equation

$$
\begin{aligned}
v_{t}= & {\left[-2 k_{0}^{2} v-v_{x x}-\kappa_{0} v^{3}-\kappa_{1} v^{5}\right]_{x x} } \\
& -c\left(v^{2} v_{x x x}\right)_{x}-d\left(v v_{x} v_{x x}\right)_{x}-e\left(v_{x}^{3}\right)_{x}+\text { higher order terms. }
\end{aligned}
$$

The coefficients in this equation are

$$
\kappa_{0}=-2\left(1-\frac{4 k_{0}^{2} \alpha}{3 R_{0}}\right),
$$

$$
\begin{gathered}
\kappa_{1}=-\frac{16 k_{0}^{2}\left[\left(3 b_{3}-10 R_{0}^{2}\right) R_{0} \alpha^{2}+2 k_{0}^{2} \alpha-R_{0}\right]}{5 R_{0}\left(r-k_{0}^{4}+3 b_{3} R_{0}^{2}-5 R_{0}^{4}\right)}-\frac{8 \alpha\left(\alpha k_{0}^{2}+R_{0}\right)}{5 R_{0}^{2}}, \quad \text { (A3) } \\
c=\frac{8 \alpha}{R_{0}}-\frac{16 k_{0}^{2}\left(R_{0}-k_{0}^{2} \alpha\right)}{R_{0}\left(r-k_{0}^{4}+3 b_{3} R_{0}^{2}-5 R_{0}^{4}\right)}, \\
d=\frac{44 \alpha}{R_{0}}-\frac{8 k_{0}^{2}\left(5 R_{0}-6 k_{0}^{2} \alpha\right)}{R_{0}\left(r-k_{0}^{4}+3 b_{3} R_{0}^{2}-5 R_{0}^{4}\right)}, \\
e=\frac{12 \alpha}{R_{0}},
\end{gathered}
$$

where

$$
\alpha=-\frac{2 k_{0}^{2} R_{0}}{r-k_{0}^{4}+3 b_{3} R_{0}^{2}-5 R_{0}^{4}},
$$

$$
R_{0}^{2}=\frac{1}{2}\left\{b_{3} \pm \sqrt{b_{3}^{2}+4\left[r-\left(v_{s}^{2}-k_{0}^{2}\right)^{2}\right]}\right\} .
$$

The Cahn-Hilliard equation (27) is obtained when $k_{0} \ll 1$.
[1] A. R. Champneys, Physica D 112, 158 (1998).

[2] J. Swift and P. Hohenberg, Phys. Rev. A 15, 319 (1977).

[3] G. W. Hunt, M. A. Peletier, A. R. Champneys, P. D. Woods, M. A. Wadee, C. J. Budd, and G. J. Lord, Nonlinear Dyn. 21, 3 (2000).

[4] P. L. Geissler and D. R. Reichman, Phys. Rev. E 69, 021501 (2004).

[5] M. Tlidi, P. Mandel, and R. Lefever, Phys. Rev. Lett. 73, 640 (1994).

[6] G. Kozyreff and M. Tlidi, Chaos 17, 037103 (2007).

[7] J. Burke and E. Knobloch, Phys. Rev. E 73, 056211 (2006).

[8] J. Burke and E. Knobloch, Phys. Lett. A 360, 681 (2007).

[9] P. D. Woods and A. R. Champneys, Physica D 129, 147 (1999).

[10] J. Knobloch and T. Wagenknecht, Physica D 206, 82 (2005).

[11] J. Burke and E. Knobloch, Chaos 17, 037102 (2007).

[12] Y. Pomeau, Physica D 23, 3 (1986).

[13] P. Coullet, C. Riera, and C. Tresser, Phys. Rev. Lett. 84, 3069 (2000).

[14] M. Beck, J. Knobloch, D. J. B. Lloyd, B. Sandstede, and T. Wagenknecht, SIAM J. Math. Anal. 41, 936 (2009).

[15] B. A. Malomed, Z. Phys. B: Condens. Matter 55, 241 (1984).

[16] M. Bestehorn and H. Haken, Phys. Rev. A 42, 7195 (1990).

[17] J. Lega, J. V. Moloney, and A. C. Newell, Phys. Rev. Lett. 73, 2978 (1994).

[18] J. Lega, J. V. Moloney, and A. C. Newell, Physica D 83, 478 (1995).

[19] K. Staliunas, Phys. Rev. A 48, 1573 (1993).

[20] S. Longhi and A. Geraci, Phys. Rev. A 54, 4581 (1996).

[21] V. J. Sánchez-Morcillo, E. Roldán, G. J. de Valcárcel, and K.
Staliunas, Phys. Rev. A 56, 3237 (1997).

[22] K. Staliunas, G. Slekys, and C. O. Weiss, Phys. Rev. Lett. 79, 2658 (1997).

[23] K. Staliunas, M. F. H. Tarroja, G. Slekys, C. O. Weiss, and L. Dambly, Phys. Rev. A 51, 4140 (1995).

[24] J.-F. Mercier and J. V. Moloney, Phys. Rev. E 66, 036221 (2002)

[25] J. M. Soto-Crespo and N. Akhmediev, Phys. Rev. E 66, 066610 (2002).

[26] A. Bergeon, J. Burke, E. Knobloch, and I. Mercader, Phys. Rev. E 78, 046201 (2008).

[27] E. Knobloch and D. R. Moore, Phys. Rev. A 42, 4693 (1990).

[28] C. Martel, E. Knobloch, and J. M. Vega, Physica D 137, 94 (2000)

[29] E. Doedel and L. S. Tuckerman, Numerical Methods for Bifurcation Problems and Large-Scale Dynamical Systems (Springer, New York, 2000).

[30] R. Montagne, E. Hernández-García, A. Amengual, and M. San Miguel, Phys. Rev. E 56, 151 (1997).

[31] M. C. Cross, P. G. Daniels, P. C. Hohenberg, and E. D. Siggia, J. Fluid Mech. 127, 155 (1983).

[32] P. E. Cladis, Y. Couder, and H. R. Brand, Phys. Rev. Lett. 55, 2945 (1985).

[33] J. D. M. Rademacher and A. Scheel, Int. J. Bifurcation Chaos Appl. Sci. Eng. 17, 2679 (2007).

[34] A. S. Landsberg and E. Knobloch, Phys. Lett. A 159, 17 (1991).

[35] J. Burke and E. Knobloch, Discrete Contin. Dyn. Syst. (to be published).

[36] E. Knobloch and M. R. E. Proctor, J. Fluid Mech. 108, 291 
(1981).

[37] J. W. Cahn and J. E. Hilliard, J. Chem. Phys. 28, 258 (1958).

[38] L. Q. Chen, Annu. Rev. Mater. Res. 32, 113 (2002).

[39] J. W. Cahn, Acta Metall. 9, 795 (1961).

[40] A. Novick-Cohen and L. A. Segel, Physica D 10, 277 (1984).

[41] J. Carr, M. E. Gurtin, and M. Slemrod, Arch. Ration. Mech. Anal. 86, 317 (1984).

[42] M. Argentina, M. G. Clerc, R. Rojas, and E. Tirapegui, Phys. Rev. E 71, 046210 (2005).

[43] D. Mermin, Rev. Mod. Phys. 51, 591 (1979).

[44] D. K. Campbell, M. Peyrard, and P. Sodano, Physica D 19, 165 (1986).

[45] C. Elphick, G. R. Ierley, O. Regev, and E. A. Spiegel, Phys. Rev. A 44, 1110 (1991).
[46] M. C. Cross and P. C. Hohenberg, Rev. Mod. Phys. 65, 851 (1993).

[47] C. L. Emmott and A. J. Bray, Phys. Rev. E 54, 4568 (1996).

[48] K. Kawasaki and T. Ohta, Physica A 116, 573 (1982).

[49] T. Nagai and K. Kawasaki, Physica A 134, 483 (1986).

[50] X. D. Sun and M. J. Ward, Stud. Appl. Math. 105, 203 (2000).

[51] J. Burke, A. Yochelis, and E. Knobloch, SIAM J. Appl. Dyn. Syst. 7, 651 (2008).

[52] Y.-P. Ma, J. Burke, and E. Knobloch (unpublished).

[53] K. Maruno, A. Ankiewicz, and N. Akhmediev, Physica D 176, 44 (2003).

[54] C. Chevallard, M. Clerc, P. Coullet, and J.-M. Gilli, Eur. Phys. J. E 1, 179 (2000).

[55] M. G. Clerc, D. Escaff, and R. Rojas, EPL 83, 28002 (2008). 